\title{
Electron Signatures of Satellite Sweeping in the Magnetosphere of Uranus
}

\author{
J. F. COOPER' AND E. C. Stone \\ California Institute of Technology, Pasadena
}

\begin{abstract}
The Voyager 2 Cosmic Ray System found large-scale macrosignatures of satellite sweeping for $\mathrm{MeV}$ electrons near the orbits of the satellites Miranda, Ariel, and Umbriel in the magnetosphere of Uranus. Due to the large magnetic inclinations of satellite orbits at Uranus, sweeping rates vary along the orbits with the Mcllwain $L$ parameter. However, no evidence was found, where expected, for fresh sweeping signatures at such positions. Although the maximal electron intensity occurs near Voyager 2's minimum $L(4.67)$ as predicted by the $\mathrm{Q}_{3}$ field model, the intensity minima in the macrosignatures show large outward displacements $\left(\leq 0.5 R_{U}\right)$ from minimum- $L$ positions of the associated satellites. These radial displacements increased with measured electron energy and at higher magnetic latitudes. Pitch angle distributions are generally more anisotropic outside the macrosignatures and more isotropic within, as determined from comparison of inbound and outbound intensity profiles at different latitudes. These anisotropy measurements provide the basis for latitudinal flux extrapolation, which when coupled with power law scaling of spectral distributions allow the calculation of phase space density profiles. The latter show local minima in the macrosignatures and are indicative of distributed electron sources in the inner magnetosphere and/or nonadiabatic transport processes such as pitch angle scattering and magnetospheric recirculation. Preliminary diffusion coefficients with values $D_{L L} \sim 10^{-7}-10^{-6} R_{S}^{2}$ and radial dependence $D_{L L} \sim L^{3}-L^{4}$ have been estimated for the macrosignatures. The low-order $L$ dependence of $D_{L L}$ is consistent with diffusion driven by ionospheric dynamo. However, quantitative modeling of radial and pitch angle diffusion is required to assess the formative processes for the macrosignatures before more physically meaningful transport parameters can be determined.
\end{abstract}

\section{INTRODUCTION}

The January 24, 1986, flyby of Voyager 2 past Uranus [Stone and Miner, 1986] revealed a complex magnetosphere with an intense and highly energetic population of trapped charged particles. Energetic electrons at energies below $10^{2}$ $\mathrm{keV}$ were detected immediately after crossing the inbound magnetopause by the Low Energy Charged Particle (LECP) [Krimigis et al., 1986] and the Cosmic Ray System (CRS) experiments; these electrons were also found by LECP at significant flux levels in the magnetotail neutral sheet after the flyby. Inward from the magnetopause, LECP and CRS found increasing intensities and energies in the trapped electron population which showed strong modulation by satellite sweeping at energies above $10^{2} \mathrm{keV}$. CRS found the highest-energy electrons, above $10 \mathrm{MeV}$, in the most intense region inside the orbit of Miranda, where only the CRS counting rates for electrons above $3 \mathrm{MeV}$ could be analyzed due to saturation effects. Within the Miranda absorption region, and beyond, the CRS response was approximately linear, and as the present analysis concludes, there was no significant background from high-energy protons.

In this paper we give a detailed account of general morphology for the observed signatures of satellite interactions with the trapped electrons. Time profiles of the more energetic electrons show deep and radially broad absorption features, hereafter designated as macrosignatures, which reflect the $60^{\circ}$ tilt angle between the dipole and rotational axes and the correspondingly large inclinations of the satel-

\footnotetext{
${ }^{1}$ Now at ST Systems Corporation, Greenbelt, Maryland.

Copyright 1991 by the American Geophysical Union.

Paper number 90JA02629.

0148-0227/91/90JA-02629\$05.00
}

lite orbits in magnetic coordinates. Correlation of the principal signatures with sweeping by Miranda, Ariel, and Umbriel was first established with the offset, tilted dipole (OTD) model for the planetary magnetic field [Ness et al., 1986], but neither the OTD model nor another dipole model derived directly from the CRS signatures [Stone et al., 1986] could give exact alignments of these signatures with positions of maximal satellite sweeping. Such positions are located near the two points in each orbit where a satellite crosses the magnetic equator, and these positions define the magnetic $L$ value [McIlwain, 1961; Stone, 1963] of the innermost drift shell reached by each satellite.

Although the sub-MeV electron signatures measured by LECP are reported to line up reasonably well with the minimum- $L$ positions of the parent satellites [Krimigis et al., 1986; Mauk et al., 1987], the CRS signatures (and those for the higher-energy LECP measurements) have intensity minima significantly outward from these positions as first determined with the OTD model. We confirm this result with the more accurate $\mathrm{Q}_{3}$ model [Connerney et al., 1987; Acuña et al., 1988], which includes dipole and quadrupole components. Furthermore, the different signature profiles found from inbound and outbound measurements at different magnetic latitudes are shown here to be associated with strong radial variations in pitch angle anisotropies in the macrosignature regions. Since no evidence is found for time dependence in the macrosignatures, particularly in that no fresh sweeping features are found near minimum $L$ or elsewhere, we conclude that the displacements reflect time-stationary characteristics of the balance between sources, radial transport, and sweeping losses. There is no indication of residual problems with the field models; indeed, $\mathrm{Q}_{3}$ gives a precise prediction of the time of maximum electron intensity with an 
error of $\Delta L \sim 0.01$, while the observed displacements are more than an order of magnitude larger than that difference.

The satellite interaction with the trapped electrons can be used to probe the nature and dynamics of sources and radial transport mechanisms which may have characteristic signatures in electron populations observed within or near the satellite sweeping regions. The fact that finite fluxes are observed in swept regions is immediately indicative of active radial transport processes and/or distributed sources. The radial extent and similarity in different satellite regions of the observed macrosignatures are strongly suggestive of near steady state conditions which produce these features as the results of time-averaged sources, radial transport, and sweeping losses.

The satellite sweeping process can be well understood in terms of available theoretical models [Chenette et al., 1986; Paonessa and Cheng, 1987; Cooper, 1990], but no evidence is found of correlation with theoretical loss rate profiles for single sweeping episodes. Indeed, we examine the one case (Umbriel's outbound macrosignature) where a narrow but deep "microsignature" was expected in the absence of diffusion, but not found, and thereby determine a local lower limit for the radial diffusion coefficient. This limit is in agreement with macrosignature-derived diffusion coefficients estimated from the macrosignature dimensions and characteristic time scales for satellite sweeping.

Our anisotropy analysis reveals large radial variations in pitch angle distributions which are characterized by more isotropic distributions within the macrosignatures. These variations may be signatures of specific source or transport processes; a specific example is the magnetospheric recirculation process [Nishida, 1976; Fujimoto and Nishida, 1990a, $b$, which invokes conventional adiabatic diffusion for inward diffusion and acceleration, pitch angle scattering, and nonadiabatic transport at low altitudes to provide a "return current" of energized electrons to the outer magnetosphere without energy loss. Recently reported observations in the Earth's magnetosphere [Baker et al., 1989] have confirmed the existence of the recirculation component, manifested as a field-aligned (i.e., dumbbell anisotropy) population of electrons above $600 \mathrm{keV}$ which shows correlation in intensity with time variations in geomagnetic indices (e.g., $K p$ ). Although the brief Voyager 2 flyby does not allow us to explore time correlations, we can ask whether recirculation might account for the electron anisotropies at Uranus and preferentially replenish the losses of more energetic electrons in the CRS response range. Alternatively, pitch angle scattering alone may give rise to the morphology of the macrosignature observations if wave-particle interactions are significant in the macrosignature regions. Other possibilities include effects of episodic diffusion [McKibben and Simpson, 1980] which might mimic observational characteristics of recirculation or other sources.

The pitch angle anisotropies may also be used, in conjunction with power law scaling for electron energy spectra, to calculate phase space density profiles for the satellite regions, where these profiles allow differentiation between effects purely due to radial gradients (i.e., intensity minimum is not a density minimum) and physical effects of active source and/or transport processes. Initial analysis of phase space densities [Stone et al., 1986] indicated that the high fluxes of $\mathrm{MeV}$ electrons in the innermost magnetosphere probed by Voyager 2 could be explained by inward diffusion and acceleration of electrons from the low-energy reservoir in the outer magnetosphere, but this analysis did not address local variations within macrosignatures. The LECP analysis [Cheng et al., 1987] shows a generally positive radial density gradient at sub-MeV energies but finds significant gradient reversals within the satellite sweeping regions, where local density minima are suggestive of additional sources within the inner magnetosphere. Since even larger deviations are found at $\mathrm{MeV}$ energies in the present work, the responsible processes apparently become even more significant at higher energies in comparison to effects of conventional inward diffusion and acceleration. Further quantitative data analysis and modeling of such processes will be subjects of future work [Selesnick and Stone, 1991].

\section{EnCOUNTER Geometry in Magnetic CoORdinates}

\section{Magnetic Field Models}

The complex magnetic geometry of satellite sweeping at Uranus requires accurate modeling of the planetary magnetic field for meaningful interpretation of the energetic trapped particle observations. Ness et al. [1986] initially defined the first model (OTD) for the planetary magnetic field of Uranus in terms of a simple magnetic dipole tilted at $60^{\circ}$ with respect to the rotational axis of Uranus and offset by $0.31 R_{U}\left(1 R_{U}=25,600 \mathrm{~km}\right)$ from the planet center. Subsequent analysis of the Voyager magnetometer data by Connerney et al. [1987] and Acuña et al. [1988] found an improved fit to a planet-centered model $\left(\mathrm{Q}_{3}\right)$ with dipole and quadrupole terms, the latter being approximately equivalent at large distances from Uranus to offsets of the dipole by 0.31 $R_{U}$ along the rotational axis (i.e., same as OTD) and by 0.15 $\boldsymbol{R}_{U}$ in the equatorial plane perpendicular to that axis.

Acuña et al. [1988] have calculated drift shell positions from $Q_{3}$ which are used in this work to determine magnetic coordinates of observed and predicted electron features. Whenever we require precise locations in magnetic coordinates, we utilize the $Q_{3}$ model results; otherwise, the OTD drift shells are used in theoretical (and usually illustrative) calculations of satellite orbits and sweeping rates. In some cases (e.g., sweeping rates) the OTD-derived quantities may be rescaled to the most appropriate $L_{\min }$ value from $\mathrm{Q}_{3}$ so that reasonable accuracy is assured.

\section{Spacecraft and Satellite Coordinates}

The improvement provided over OTD by the $\mathrm{Q}_{3}$ model in determination of spacecraft trajectory in magnetic coordinates is evident in Figure 1, where the calculated time profile of the spacecraft's local position with respect to the drift shell parameter $L$ shows divergences $\Delta L \leq 1$ between the $\mathrm{Q}_{3}$ and OTD models. The time-dependent positions in $L$ of the satellites Miranda, Ariel, and Umbriel are also shown from $\mathrm{Q}_{3}$ in the same time period. The large dipole tilt is particularly evident in the periodic excursions of the satellite positions in a broad region at $L \geq L_{\text {min }}$.

The $Q_{3}$ model allows accurate calculation of drift shell locations for correlation with localized trapped particle features, while OTD facilitates tractable numerical calculations of longitudinally averaged parameters for satellite sweeping [cf. Paonessa and Cheng, 1987; Cooper, 1990]. The relation between the two models is further shown in 


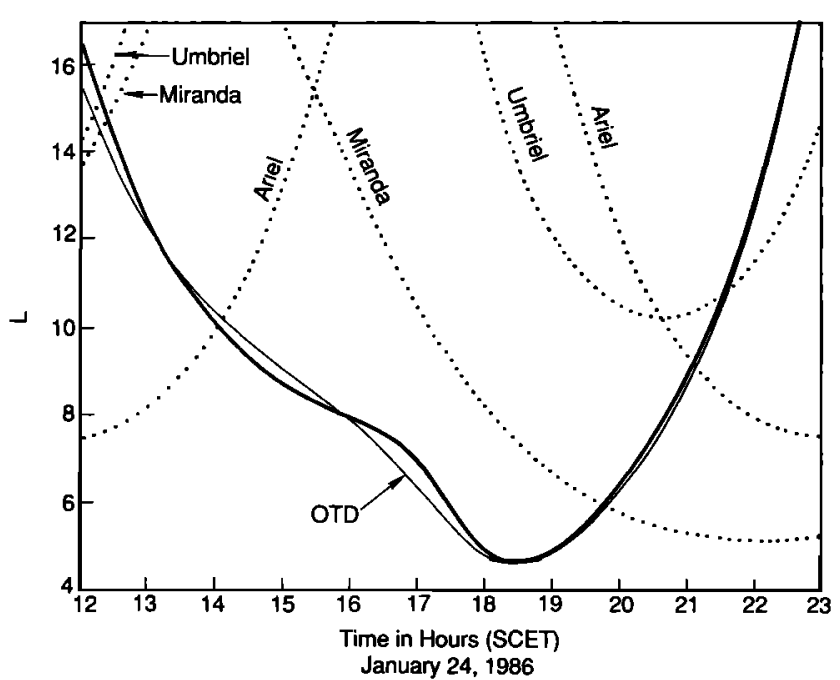

Fig. 1. Drift shell locations in $L$ of Voyager 2 and the satellites Miranda, Ariel, and Umbriel from the $\mathrm{Q}_{3}$ field model [Connerney et al., 1987; Acuña et al., 1988]. The OTD [Ness et al., 1986] trajectory profile for Voyager 2 is also shown for comparison.

Figure 2, where the ratio $B / B_{0}$ ( $B$ is local magnetic field magnitude, and $B_{0}$ is the equatorial value at the same $L$ ) is plotted versus $L$ for the orbits of the satellites from each model. With OTD the magnetic orbit of each satellite can be characterized approximately by a single curve in Figure 2, except for minor deviations due to orbital eccentricities and inclinations, while the $Q_{3}$ curves show a range of variation centered on the corresponding OTD curves for each satellite. The $Q_{3}$ variation can be understood in terms of an offset

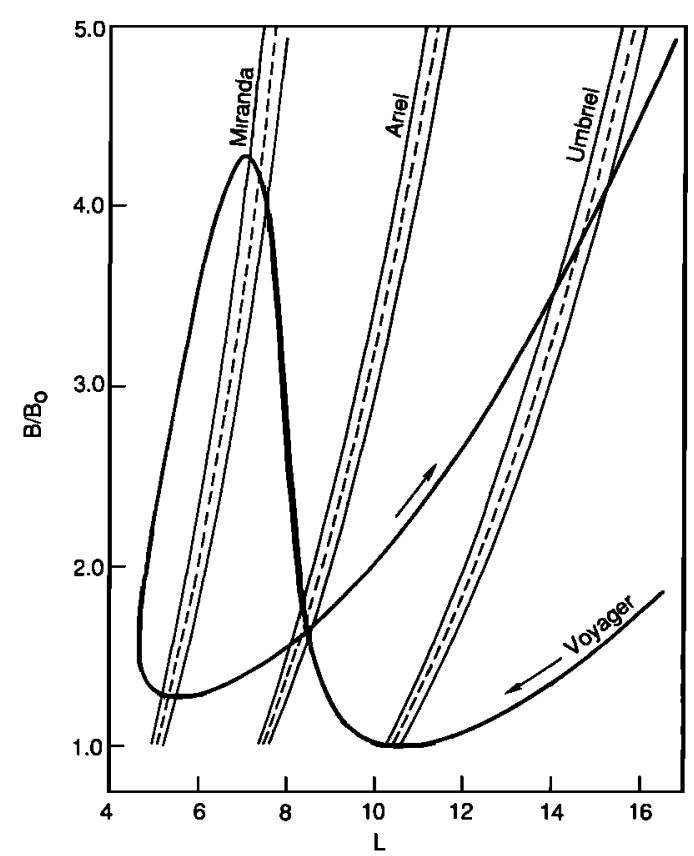

Fig. 2. $\quad B$ and $L$ coordinates of Voyager 2 and the satellite orbits with $B$ normalized to the equatorial value $B_{0}$. The solid curves are from $Q_{3}$, and the variable orbits of the satellites are delimited by pairs of curves. The dashed curves show the OTD coordinates for the satellites and correspond to longitudinal averages of the $Q_{3}$ curves. in the Uranographic equatorial plane of the equivalent dipole center for $\mathrm{Q}_{3}$; the magnetic orbit is closer to the Uranographic center at some longitudes than at others.

The magnitude of the equatorial offset affects the range of variation in $L_{\min }$ with respect to the OTD value for each satellite. Since the satellite orbit crosses the plane of the OTD equator twice, approximately at the same distance from the dipole center, the OTD minimum $L$ of each satellite is uniquely defined by $L_{\min }=\left(a^{2}+z_{0}^{2}\right)^{1 / 2}$, where $a$ is the semimajor radius of the satellite orbit and $z_{0}$ is the OTD offset on the Uranographic rotational axis (both in units of $\left.R_{U}\right)$. The OTD $L_{\min }$ values for Miranda, Ariel, and Umbriel are $5.083,7.463$, and 10.39 , respectively.

In contrast, the $0.15-R_{S}$ equatorial offset in $Q_{3}$ produces a bimodal dispersion $\Delta L \sim \pm 0.15$ in the minimum- $L$ values for alternate crossings of the minimum- $B$ equator at different Uranographic longitudes. Table 1 lists the two most recent minimum- $L$ values for each satellite in the macrosignature regions sampled during the Voyager 2 encounter. In general, the intensity minima in the regions were found outside the corresponding $L_{\min }$ positions in the table. Only in the case of Umbriel did a satellite pass through $L_{\min }$ in the interim between inbound and outbound observations and thereby afford an opportunity for detection of recent sweeping effects (which were not observed, as discussed in section 4).

The breadth and displacement (see sections 3 and 4) of the satellite macrosignatures, and the absence of satellite microsignatures [Stone et al., 1986], preclude precision testing of the planetary field model for Uranus with charged particle data, but we can establish a single-point test utilizing the time of maximum electron intensity registered by CRS and the time of Voyager 2's minimum $L$ as predicted by the two models. The maximum intensity for CRS electrons at Uranus was found at $1836( \pm 3 \mathrm{~min})$ SCET (spacecraft event time) (see the appendix). The corresponding value of $L$ from $\mathrm{Q}_{3}$ is $4.68 \pm 0.01$ and is very near the calculated minimum $L$ of 4.666 for Voyager 2 at 1829:11 \pm 48 s SCET from $Q_{3}$ (J. E. P. Connerney and M. H. Acuña, private communication, 1987). In contrast, the OTD field model predicts the same minimum $L$ for Voyager 2 at an earlier time of 1812:47 $\pm 24 \mathrm{~s}$ SCET.

In the absence of strong sweeping effects, the correlation of maximum intensity with minimum $L$ of Voyager 2 is expected from the inward diffusion and acceleration of energetic electrons which increase the integral intensities of accelerated electrons above fixed energy thresholds as $L$ decreases. The fact that maximum intensity was observed 7 min after reaching the $Q_{3}$ minimum $L$ is consistent with an expected latitudinal gradient (see section 5). However, the $L$ value at the flux maximum is only 0.01 greater than that at minimum $L$. This observation provides a one-point test of $Q_{3}$ values for $L$ which were calculated with the approximation that drift shell splitting is negligible [Acuña et al., 1988]. Since the effects of high-order field components decline at larger distances from Uranus, we assume that the accuracy of drift shell positions calculated from $\mathrm{Q}_{3}$ is better than $\Delta L \sim 0.01$ in the macrosignature regions where we localize positions of intensity minima in $L$.

\section{CRS Response to Pitch Angle Distributions}

The nature of trapped particle distributions is such that local fluxes vary in a predictable fashion as a function of 
TABLE 1. Satellite Absorption Signature Data

\begin{tabular}{|c|c|c|c|c|c|c|c|}
\hline \multirow[b]{2}{*}{ Satellite } & \multirow[b]{2}{*}{$\mathrm{IB} / \mathrm{OB}^{a}$} & \multicolumn{2}{|c|}{ Satellite $L_{\min }$} & \multicolumn{4}{|c|}{ Intensity Minima $^{b}$} \\
\hline & & Recent & Previous & $E, \mathrm{MeV}$ & $L$ & $B / B_{0}$ & SCET \\
\hline Umbriel & IB & 10.522 & 10.311 & $\geq 1.1$ & $10.74 \pm 0.03$ & 1.00 & $1340: 00 \pm 48 \mathrm{~s}$ \\
\hline Ariel & IB & 7.319 & 7.542 & $\geq 1.1$ & $7.84 \pm 0.01$ & 3.09 & $1612: 00 \pm 48 \mathrm{~s}$ \\
\hline Miranda & IB & 4.929 & 5.162 & $\geq 1.1$ & $5.41 \pm 0.03$ & 2.86 & $1742: 50 \pm 48 s$ \\
\hline Miranda & IB & 4.929 & 5.162 & $\geq 3.1$ & $\ldots$ & $\cdots$ & $\ldots$ \\
\hline Miranda & $\mathrm{OB}$ & 4.929 & 5.162 & $\geq 1.1$ & $5.28 \pm 0.01^{c}$ & 1.28 & $1918: 51 \pm 60 \mathrm{~s}$ \\
\hline Miranda & OB & 4.929 & 5.162 & $\geqq 2.7$ & $5.34 \pm 0.03^{c}$ & 1.28 & $1921: 44 \pm 60 \mathrm{~s}$ \\
\hline Miranda & OB & 4.929 & 5.162 & $\geq 3.1$ & $5.40 \pm 0.04$ & 1.27 & $1924: 08 \pm 96 \mathrm{~s}$ \\
\hline Ariel & OB & 7.319 & 7.542 & $\geq 1.1$ & $7.70 \pm 0.04^{c}$ & 1.50 & $2034: 27 \pm 60 \mathrm{~s}$ \\
\hline Umbriel & OB & 10.275 & 10.522 & $\geq 1.1$ & $\cdots$ & $\cdots$ & \\
\hline
\end{tabular}

These $L$ and $B / B_{0}$ values from $Q_{3}$ were supplied by the Voyager magnetometer group [Connerney et al., 1987; Acuña et al., 1988; M. Acuña and J. E. P. Connerney, private communication, 1987]. Three dots indicate that the local intensity minimum was not observed.

${ }^{a}$ Inbound (IB) and outbound (OB).

${ }^{b}$ Determined from 6-s and 96-s counting rates.

${ }^{c}$ Data gap contributes to uncertainty in position of minimum.

local field $B$ and the local pitch angle $\alpha$ for some types of pitch angle distributions. Integrated over all pitch angles, the omnidirectional flux varies as $J(L, B) \propto\left(B_{0} / B\right)^{n}$ along a field line of constant $L$ for "pancake" pitch angle distributions of the form $j(L, B, \alpha) \propto \sin ^{2 n} \alpha / B^{n}$. During most of the encounter the omnidirectional approximation is appropriate, considering the wide-angle response of most CRS detectors (see section 5). The large inbound changes in $B / B_{0}$ during traversal of the Ariel and Miranda regions would therefore produce the largest flux variations for nonzero values of $n$. In the absence of significant time variations the omnidirectional fluxes near $L \sim 8.5$ inbound and outbound would have been equal, while the inbound and outbound fluxes measured at other $L$ values might have shown large relative variations dependent on the local anisotropies. Moreover, these anisotropies can be estimated wherever omnidirectional fluxes are measured at different latitudinal positions, as discussed in section 5 .

In interpreting time profiles for CRS counting rates it is of interest to know when directional response may become significant. In this case a highly directional sensor would respond to the directional flux $j(L, B, \alpha)$. Figure 3 shows time profiles of local pitch angles in OTD and $Q_{3}$ coordinates for the pointing directions of symmetry axes for three CRS sensor heads used in the present analysis: the electron telescope (TET), high-energy telescope I (HET-I), and highenergy telescope II (HET-II) (see the appendix). The irregularity in the inbound profiles may be attributed in part to several spacecraft roll maneuvers (see Figure 4). The OTD and $Q_{3}$ profiles for each sensor differ by less than $10^{\circ}$, a negligible difference in view of the wide-angle responses (see section 5). Note that the differences are least outbound, when the differences in OTD and $\mathrm{Q}_{3} L$ values of Voyager 2 (see Figure 1) are also minimal. Since such differences are insignificant with respect to other uncertainties in our anisotropy analysis (e.g., the parameterization of the pitch angle distributions with respect to two-point measurements on each drift shell), we follow the LECP authors [Mauk et al., 1987] in using OTD pitch angles for the present anisotropy analysis in section 5 .

\section{Electron Measurements}

\section{Overview of Time Profiles}

Figure 4 shows the large dynamic range of CRS and LECP response to electrons at energies of $\leq 0.1 \mathrm{MeV}$ to above 10
$\mathrm{MeV}$ in the inner magnetosphere of Uranus. The energy thresholds (1.1-10 MeV) for CRS counting rates are calculated by the bow tie approximation [Fillius and McIlwain, 1974; Van Allen et al., 1974] for response of single, shielded detectors to electrons. Definition of the rates and other instrument details are given in the appendix. The radial coverages of the electron measurements decrease with increasing energy as the measured intensities fall to background levels, such that the highest-energy electrons $(\geq 10$ $\mathrm{MeV}$ ) were detected by CRS only inside the orbit of Miranda. The relatively small electron detectors used in the LECP experiment allow more linear counting rate measurements at the highest electron intensities for energies $\leq \mathbf{0 . 8}$ $\mathrm{MeV}$ but have limited radial coverage at higher energies due to low count rates. The most nearly comparable LECP and CRS electron thresholds are at $853 \mathrm{keV}$ and $1.1 \mathrm{MeV}$,

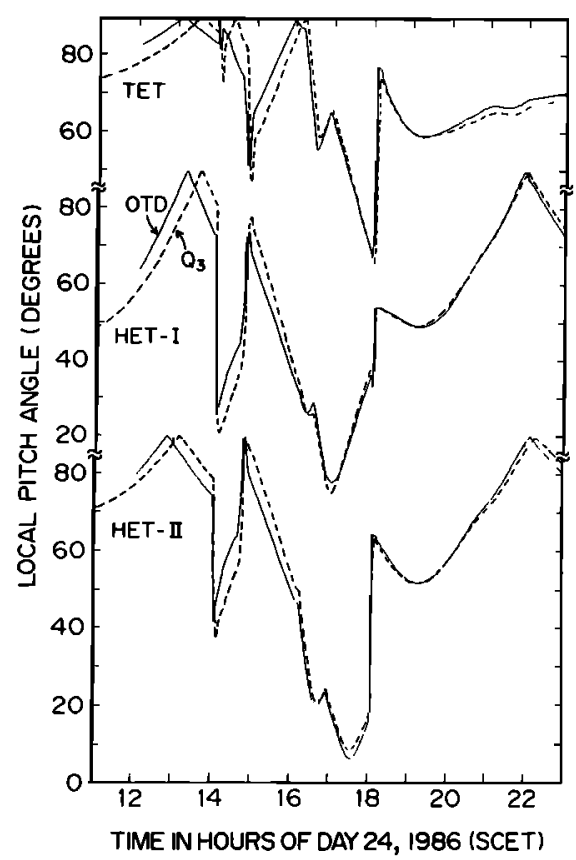

Fig. 3. Comparison of time profiles for local pitch angles of the boresight pointing directions for the TET, HET-I, and HET-II telescopes in the Voyager 2 Cosmic Ray System (CRS) (see the appendix). Solid and dashed curves are for pitch angles calculated with the OTD and $Q_{3}$ field models, respectively. 


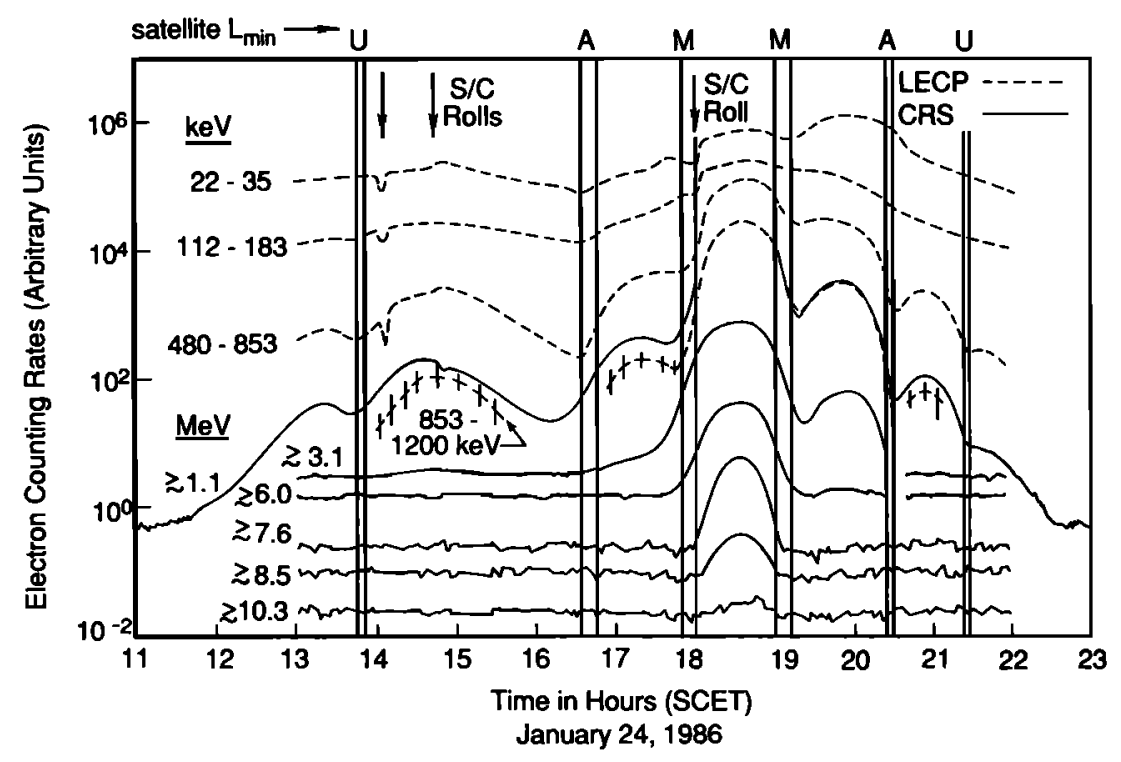

Fig. 4. Integral counting rates versus spacecraft event time (SCET) in hours of day 24, 1986, for the following electron energy thresholds and associated CRS detectors: $1.1 \mathrm{MeV}$ (D1L), 3.1 MeV (C4H), 6.0 MeV (C3H), 7.6 MeV (C4L), 8.5 MeV (C2L), and 10.3 MeV (C3L). In comparison we also show LECP counting rates for 22- to 35-keV, 112to $183-\mathrm{keV}$, and 480 - to $853-\mathrm{keV}$ electrons [Mauk et al., 1987]. All counting rates in this figure were renormalized for comparison of time profile shapes, and the ordinate scale values do not reflect the actual count rate levels. For reference the CRS counting rates at peak intensities near 1830 SCET are $4.8 \times 10^{4}, 1.2 \times 10^{4}, 1.8 \times 10^{3}, 3.3 \times 10^{2}, 5.0 \times 10^{1}$, and $2.0 \times 10^{1} \mathrm{cps}$, respectively, and the LECP rate values can be found in Figure 8 of Mauk et al. [1987]. Pairs of vertical lines mark spacecraft crossing times at the two most recent minimum- $L$ positions of Miranda (M), Ariel (A), and Umbriel (U). Vertical arrows mark the onset times for spacecraft roll maneuvers.

respectively, but only the latter provides complete radial coverage of the satellite signatures in the $\mathrm{MeV}$ range. The CRS data are shown without corrections for counting dead time, which are discussed in the appendix and would be minimal in regions of interest (i.e., satellite macrosignatures) for this paper. Our assessment of possible proton components in the nominal electron counting rates is also discussed in the appendix.

The satellite sweeping signatures are major components in the observed variations of the electron counting rates in Figure 4. Except for small data gaps the radial coverages of the signatures for Miranda, Ariel, and Umbriel are complete at $\geq 1.1-\mathrm{MeV}$ energies in the CRS data and for thresholds at and below $480 \mathrm{keV}$ in the LECP data. The CRS counting rates at higher energy thresholds, $\geq 3.1 \mathrm{MeV}$, are clearly more restricted in radial coverage by high background levels. In some cases the signatures are not clearly resolved as local minima but appear only as transitions in the local radial intensity gradients, such as near 2126 SCET for the outbound Umbriel signature. Near the time corresponding to the local minimum of the $480-$ to $853-\mathrm{keV}$ signature for Miranda near 1750 SCET, there is a minimum in the $\geq 1.1$ $\mathrm{MeV}$ rate, slightly deeper than that of the $480-$ to $853-\mathrm{keV}$ profile, but not in the $\geq 3.1-\mathrm{MeV}$ rate, even though the latter rate is well above background at that time. In general, the local minima of the CRS signatures are better resolved in the inbound profiles, due to the more expanded scale of $L$ in time and the lesser impact of data gaps. The HET-I (see Table $\mathrm{Al}$ in the appendix) counting rates for $\geq 3.1-\mathrm{MeV}$ and $\geq 6.0-\mathrm{MeV}$ electrons have some unfortunate data gaps in the outbound Miranda and Ariel signatures, which were accentuated by the 3-min gaps due to cycling of these and other energy thresholds in the HET-I sensor.

\section{Dependence on Orientation and Latitudinal Position}

Variations in spatial orientation of the charged particle instruments (see Figure 3) should be considered in the interpretation of profiles in Figure 4. The wide-angle response to $\mathrm{MeV}$ electrons makes this effect relatively less significant for CRS, in comparison to the more collimated response of the LECP instrument at sub-MeV energies. The three roll maneuvers indicated in Figure 4 did not occur in the CRS signature regions and showed a perceptible effect only for the one near 1445 SCET. The sharp step near 1802 SCET in the lowest-energy profiles from LECP did result from the associated roll maneuver and had no relation to satellite sweeping.

The omnidirectional intensity of trapped particles varies strongly as a function of latitudinal position in some regions of the magnetosphere. The inverse correlation of the observed integral counting rates to $B / B_{0}$ is shown as a function of $L$ in Figure 5. The variation in electron rates near the inbound-outbound crossover at $L \sim 8.5$ indicates a strong dependence on $B / B_{0}$ and a significant degree of stability in trapped electron distributions for the intervening 6 hours. The increase in $B / B_{0}$ by a factor of 3 from 1445 SCET to 1639 SCET partially accounts for the declines in all counting rates during this period, although the order of magnitude decreases at energies above $480 \mathrm{keV}$ in Figure 4 are also partially due to satellite sweeping by Ariel. In comparison the outbound Ariel signature appears at a relatively constant value of $B / B_{0} \sim 1.5$ and may better represent the radial structure in the Ariel region.

The lack of recognizable intensity minima in some signatures is correlated with spacecraft position at large values of $B / B_{0}$, although energy dependence is also involved. For 


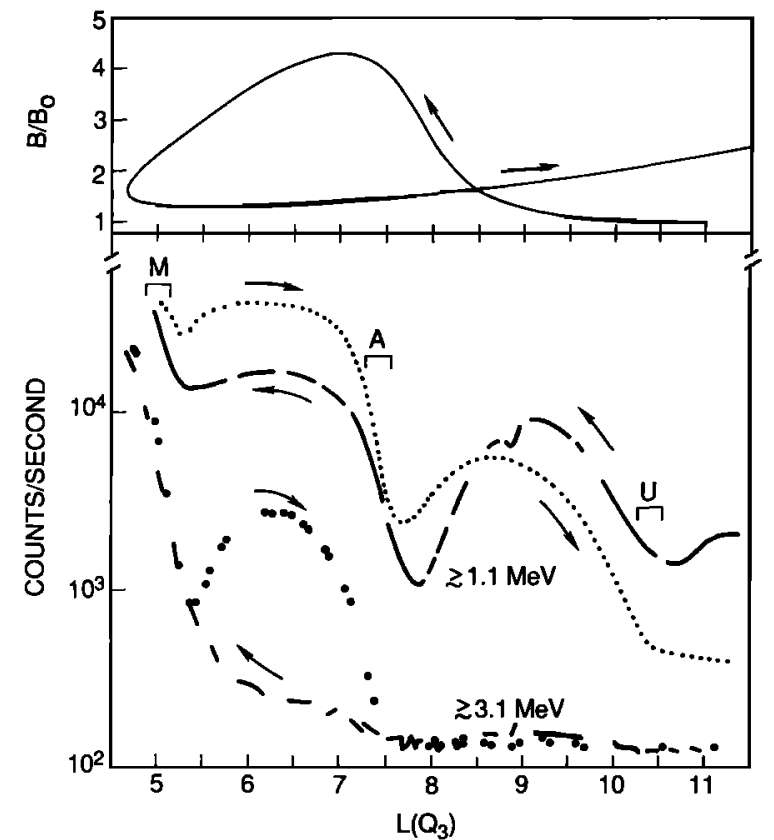

Fig. 5. (Top) Normalized field magnitude $\left(B / B_{0}\right)$ at Voyager 2 as function of drift shell parameter $L$ from $Q_{3}$ field model. Arrows indicate inbound and outbound portions of trajectory. (Bottom) Integral counting rates versus $L$ for $\geq 1.1-\mathrm{MeV}$ (D1L) and $\geq 3.1$ $\mathrm{MeV}(\mathrm{C} 4 \mathrm{H})$ electrons. The radial ranges of the two most recent minimum- $L$ positions are delimited for Miranda (M), Ariel (A), and Umbriel (U). Note the inverse correlation of counting rates with $B / B_{0}$. A time of 6 hours elapsed between inbound and outbound traversals of the crossover point near $L \sim 8.5$.

example, there is no minimum in the outbound Umbriel signature at $B / B_{0} \geq 2$, but the nearly equatorial (i.e., $B / B_{0} \sim$ 1.0) observation inbound shows a significant minimum. Although minima in other satellite signatures do appear both inbound and outbound for $\geq 1$.1-MeV electrons, the higherenergy profiles for $\geq 3$.1-MeV electrons show a large inbound-outbound difference in Figure 5 , again correlated with the relative inbound-outbound values of $B / B_{0}$, where the deep minimum outbound did not appear in the inbound profile. The convergence of the higher-energy profiles at $L \leq$ 5.4 indicates a large radial variation in electron anisotropy in the Miranda sweeping region for these energies, tending toward more isotropic fluxes at and inward of the signature minimum.

\section{Energy Dependence}

The energy dependence of satellite sweeping is evident in the very different levels of satellite-associated modulation in the profiles of Figure 4 for electrons below $183 \mathrm{keV}$ and above $480 \mathrm{keV}$. The relatively small modulations in the low-energy profiles (exceptions for $22-35 \mathrm{keV}$ at $1900-2050$ SCET are noted below) are due primarily to changes in spacecraft orientation and latitudinal position, while the increasingly deeper modulations above $480 \mathrm{keV}$ are due to sweeping. Theoretical calculations [Chenette et al., 1986; Paonessa and Cheng, 1987; Cooper, 1990] indicate that increased absorption (approximately proportional to electron kinetic energy) should occur at energies above $10^{2} \mathrm{keV}$ as observed. Little absorption is expected in the lowestenergy LECP electron channel, where sweeping times (see section 6) should be very long, due to longitudinal drift resonance with the orbital motion of satellites in the corotating magnetic field frame.

The apparent exception in the observations to the expected energy dependence is the modulation in the 22- to $35-\mathrm{keV}$ profile at 1900-1920 SCET when none was evident at $112-183 \mathrm{keV}$. This modulation is tentatively attributed (B. H. Mauk, private communication, 1990) to the presence in the inner magnetosphere of an hot Maxwellian component in the electron spectrum below $10^{2} \mathrm{keV}$. Below $10 \mathrm{keV}$ the Voyager 2 Plasma Science Experiment (PLS) found evidence of spacecraft charging at 1920-2150 SCET which was thought to be caused by enhancements of fluxes above 10 $\mathrm{keV}$ [Sittler et al., 1987]; this enhancement is evident in the 22- to 35-keV profiles of Figure 4 at 1930-2030 SCET. It is possible that this enhancement is associated with Voyager 2 's outbound approach near the magnetic equator at $L \sim 6$ during this period and/or with day-night asymmetries in the magnetospheric hot plasma. In any case the time structure in the 22- to 35-keV profile at 1900-2050 SCET is unlikely to be associated with satellite sweeping.

\section{Macrosignature Positions}

In this section we will discuss the relative positions of minimum measured intensities in the macrosignatures with respect to the minimum- $L$ positions of the associated satellites. It is clearly evident in Figures 4 and 5 that the signatures with recognizable intensity minima appear radially outside the range of minimum- $L$ positions for the corresponding satellites. Figures 6-11 show expanded profiles of the signatures as measured by CRS counting rates with maximum time resolution of $6 \mathrm{~s}$. The vertical arrows mark times where the spacecraft crossed the two most recent minimum- $L$ positions of a satellite orbit in drift shell coordinates. The prior times at which the satellite was at these positions are given in Table 1, and the differences in hours from the spacecraft times are shown above the minimum- $L$ arrows. These time differences provide a measure of the signature "ages" since the times of recent absorption. Our estimates of the approximate times for detection of intensity minima are also marked by arrows.

\section{Umbriel}

The inbound Umbriel signature in Figure 6 is particularly interesting because it falls within a time period when the spacecraft was very near the minimum- $B$ equator at $B / B_{0} \leq$ 1.01. During this period, CRS sampled the entire electron population on the local drift shells, including the equatorially trapped component. The inbound 6-s counting rates for $\geq 1.1-\mathrm{MeV}$ electrons (TAN counting rate as defined in the appendix) prior to 1339 SCET fall rapidly into a broad minimum region which extends from the transition in radial gradient near that time until the onset of a data gap at 1344-1349 SCET. Although the radial width of the broad minimum region may well extend into the data gap, this region is clearly not confined to the minimum- $L$ range of Umbriel but extends outward $0.2-0.4 R_{U}$ from the two most recent minimum- $L$ positions of Umbriel. Due to the significant scatter of the 6-s points the intensity minima for this signature and some others are determined with 96-s averages. This macrosignature is the only one out of five with recognizable intensity minima (not found for outbound Um- 
briel macrosignature) in which the observed minimum was closest to, but still outside, the most recent minimum $L$ of the parent satellite. Note that no significant feature appears in the rising inward profile at the earlier minimum- $L$ position.

A pronounced intensity minimum does not appear in Figure 7 for the outbound signature, which shows instead a radial gradient transition at $L \sim 10.3-10.4$ into a relatively flat profile extending well beyond the broad minimum of the inbound signature. The absence of the outbound minimum is probably a latitude effect and cannot be associated with effects of recent sweeping as suggested by Acuña et al. [1988], although Umbriel did reach its most recent minimum $L$ at 10.275 only 46 min before Voyager 2 arrived at that position. Due to the large separation in magnetic longitude, no fresh drift shadow or "microsignature" of Umbriel would have appeared in the 1- to $2-\mathrm{MeV}$ electrons measured by CRS, except in a narrow region at $L \sim 10.28-10.32$.

In Figure 7 we also show that detectable drift shadows at 1.1-1.5 MeV would have been produced as Umbriel moved inward toward its minimum $L$, while shadows for higher energies would have been produced during its outward movement thereafter. In this region a deep microsignature might have been observed, since the drift shadow would have affected measurable electrons at all local pitch angles, but it appears to have been dispersed by radial diffusion [e.g., Van Allen et al., 1980], as discussed further in section 6. Outside the drift shadow region the macrosignature profile

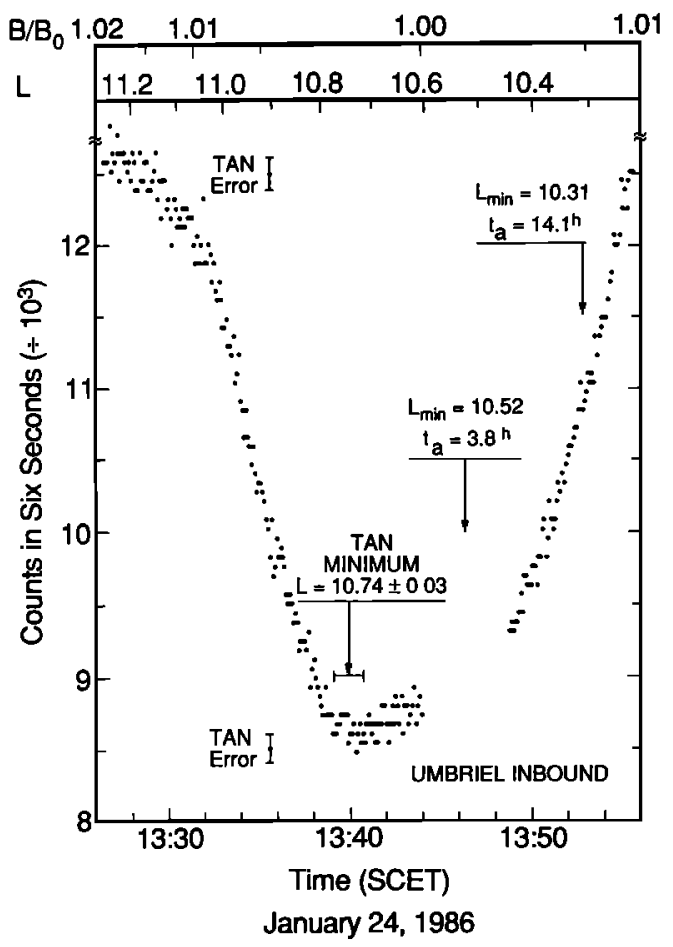

Fig. 6. Expanded time (SCET) profile from 6-s counts of TAN for $\geq 1.1-\mathrm{MeV}$ electrons in the inbound macrosignature of Umbriel. The $1 \sigma$ error limits are shown at high and low count levels. Vertical arrows mark the two most recent minimum- $L$ drift positions of Umbriel and the approximate time $( \pm 48 \mathrm{~s})$ of minimum intensity in the macrosignature. The times $t_{a}$ since Umbriel was last at the minimum- $L$ positions are given with the corresponding $L$ values. The top scales give local values of $L$ and $B / B_{0}$ corresponding to the time axis.

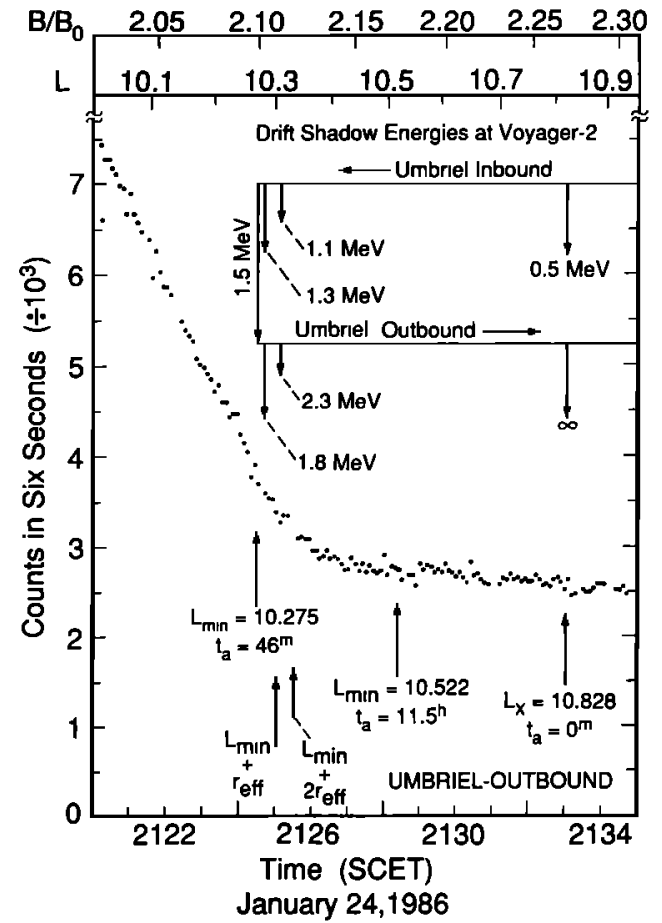

Fig. 7. Expanded time (SCET) profile for outbound macrosignature of Umbriel. Radial positions are marked with arrows for Voyager 2's expected intercept of drift shadows formed at selected electron energies by Umbriel's most recent passage toward and away from minimum $L$. Shadows for energies below $1.5 \mathrm{MeV}$ formed during the satellite's inward passage, and higher-energy shadows formed later as it moved outward. At $L=10.286$ the 1.3-MeV shadow would have drifted westward (i.e., in the opposite direction to corotation) over a magnetic longitude (OTD) interval of $277^{\circ}$ in a time of about 53 min before reaching Voyager 2. Due to the satellite's large diameter much of the observable shadow region was swept simultaneously, while the shadow intercepts shown here were calculated with respect to sweeping at the instantaneous position of the satellite center. At $L=10.286$ the effect of finite satellite diameter gives the shadow a finite energy band in the range of 1.1-1.6 MeV at the time of intercept. In this band the shadow affects about $80 \%$ of the integral electron flux above $1.1 \mathrm{MeV}$.

is most likely a quasi-stable feature formed by radial transport and absorption over many sweeping episodes.

\section{Ariel}

The inbound (Figure 8) and outbound (Figure 9) signatures for Ariel both have recognizable minima with displacements of $\Delta L \sim 0.4-0.5$ from the same minimum- $L$ position for Ariel. The minimum intensity point in the outbound signature has been estimated within the data gap by extrapolation of the well-defined profiles on each side of the gap, the minimum being located closer to the inner edge at $L=7.76$ than to the outer edge. The larger displacement of the inbound minimum is correlated with a larger value of $B / B_{0}$ (refer to Table 1). The small difference from that found inbound is clearly not so marked as might be expected (see section 5 ) from the large differences in $B / B_{0}$, especially considering the large inbound-outbound asymmetry found in the Umbriel signatures at lower values $B / B_{0} \leq 2$, and indicates a more isotropic electron population in the Ariel microsignature region (see section 5). The large time intervals (5.3 and 9.0 hours) since Ariel was at the most recent 


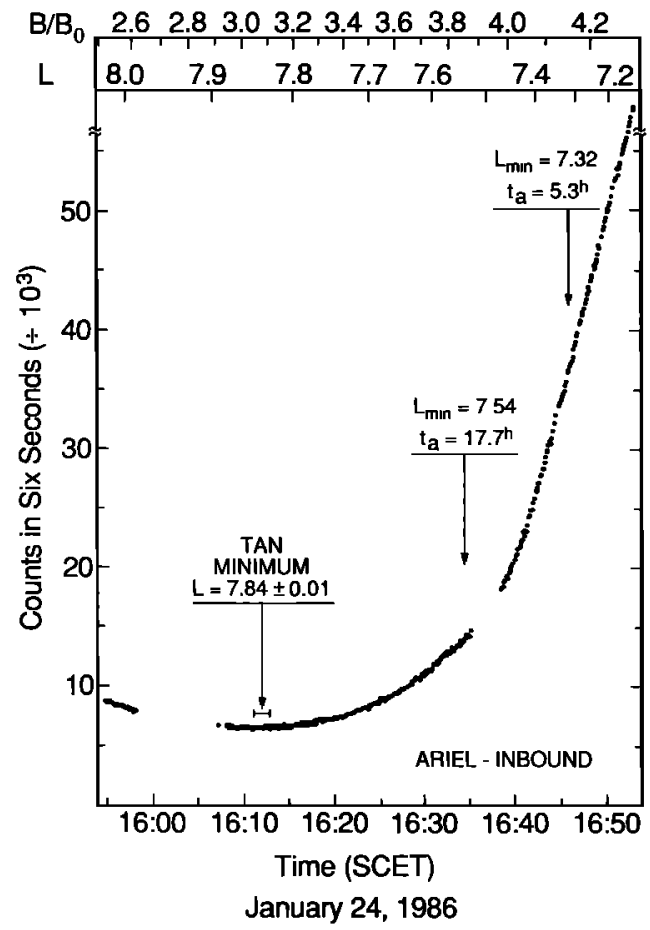

Fig. 8. Expanded time (SCET) profile for inbound macrosignature of Ariel. Refer to caption for Figure 6.

minimum $L$ (7.32) explain the absence of localized signatures at those positions inbound and outbound, particularly since diffusion dispersed the otherwise observable Umbriel drift shadow on a much shorter time scale.

\section{Miranda}

The high electron intensities in the Miranda signatures provide the only opportunity for study of the dependence of signature shapes on electron energies in the CRS energy range. The various integral count data (see Table $A l$ in the appendix) in Figure 10 and Figure 11 have electron thresholds near either 1 or $3 \mathrm{MeV}$ and excellent statistics due to high counting rates. The data with $3 \mathrm{MeV}$ thresholds are all well above cosmic ray background levels by an order of magnitude in the Miranda signatures but are backgroundlimited in the other signatures. Again, we find no features at the most recent minimum $L$ (4.93) which could be remnants of the last sweeping episode many hours earlier.

The inbound signature shows a striking change in profile between the two threshold levels. The well-defined minimum near $L \sim 5.4$ for $\geq 1$.1-MeV electrons is clearly not present in the profiles for the higher thresholds which continue to decline in the region at $L \geq 5.4$. Despite differences in geometric response factors and pointing directions the declines in the $\geq 3-\mathrm{MeV}$ profiles from the $\mathrm{C} 4 \mathrm{H}, \mathrm{B} 2 \mathrm{~L}$, and $\mathrm{D} 1 \mathrm{H}$ detectors (respectively from sensors HET-I, HET-II, and TET) are similar. The nearly identical profiles for B2L and D1H inbound apparently arise from very similar pointing directions, although we do not have detailed models of directional response for rates, such as these, with low efficiencies for response to electrons above the nominal energy thresholds in Table A1. Similarity also appears in the profiles for $\geq 1.1-\mathrm{MeV}$ electrons from the D1L (TAN rate from TET) and B2H (HET-I) detector data. We therefore conclude that the principal difference between profiles for different thresholds arises from the energy dependence in the time-averaged absorption signatures. Broader regions of significant absorption with increasing energy would be consistent with increased efficiency for satellite sweeping at higher energies. The absence of a minimum in the $\geq 3-\mathrm{MeV}$ profiles inbound is correlated, as before in the Umbriel signatures, with measurements at large values of $B / B_{0}$ and with radial variations in anisotropy distributions for steady state absorption features.

The inbound-outbound difference in the Miranda signatures is large for the $\geq 3-\mathrm{MeV}$ electrons but not evident for the lower thresholds, which have similar profiles inbound and outbound. Although dead time corrections for profiles from the D1 detector are relatively more important at the higher intensities outbound, the similar shapes of the D1L (TAN) and B2H profiles suggest that these corrections are not large at 6-s rates near and below $10^{5} / 6 \mathrm{~s}$. The strong energy dependence of the outbound profiles is probably not an artifact of nonlinear rate response at high intensities. Broadening of the satellite sweeping regions at higher energies and magnetic latitudes might account for the inboundoutbound asymmetry at the higher energies (see section 5).

The data gap in the outbound Miranda data makes the location of the signature minima uncertain, but the 6-s count rates on each edge of the gap allow us to set limits on the position of the minima. The lower threshold data show lower rates at the inner edge of the gap at $L \sim 5.27$. The higher threshold data show lower counts on the outward edge at $L \sim 5.34$ and indicate an energy-dependent effect in the observed radial locations of intensity minima which is further indicative of broader regions of significant sweeping by Miranda at higher energies. The data from 96-s averages

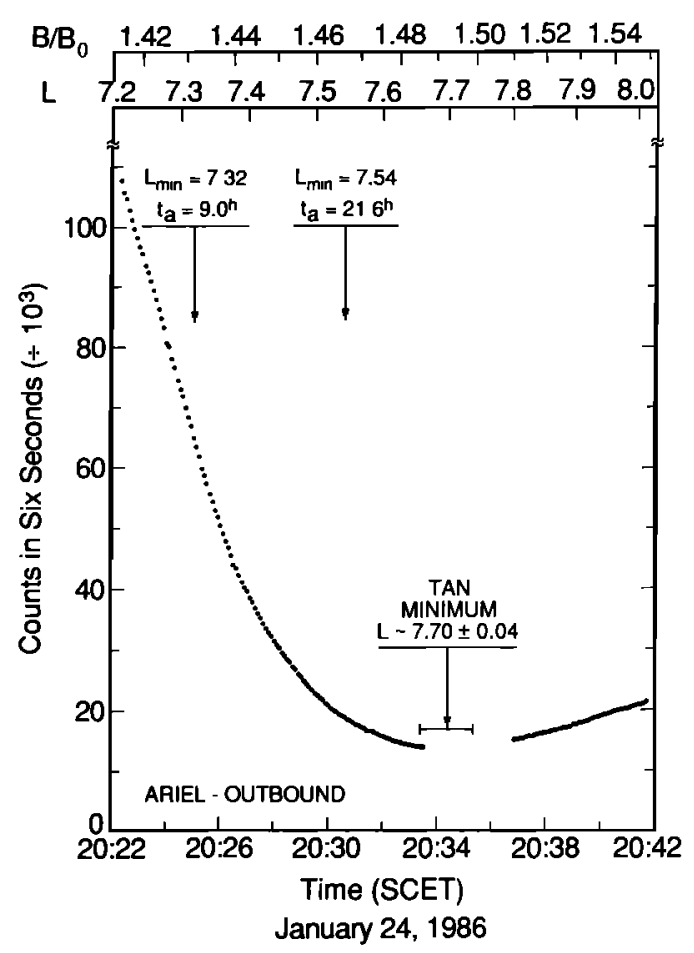

Fig. 9. Expanded time (SCET) profile for outbound macrosignature of Ariel. Refer to caption for Figure 6. 
show the same effect, although the cycling of HET-I and the $\mathrm{C} 4$ detector (see the appendix) result in a poorer definition of the minimum for the $\mathrm{C} 4 \mathrm{H}$ rates. The nearly equal values of two consecutive points for $\mathrm{C} 4 \mathrm{H}$ near $L \sim 5.40$ are consistent, however, with a minimum location within or outward from the gap.

\section{Comparison With Sub-MeV Observations}

The LECP experiments [Mauk et al., 1987] have reported that the intensity minima in satellite signatures at sub-MeV energies ( $\geq 22 \mathrm{keV}$ ) are (p. 15,292) "reasonably well-aligned with the average satellite minimum $L$ shell positions" as determined from the $\mathrm{Q}_{3}$ field model. The fact that this is not confirmed at $\mathrm{MeV}$ energies motivates a closer look at the published LECP data. Figure 8 in the paper of Mauk et al. shows the time-intensity profiles of their electron counting rates with thresholds from $22 \mathrm{keV}$ to $853 \mathrm{keV}$. Some of these rates are reproduced in our Figure 4. Although the absorption features are indeed generally aligned with satellite minimum- $L$ shell positions, closer examination indicates that the counting rate minima lie outside the most recent minimum- $L$ positions of Miranda and Ariel. In the case of the inbound Umbriel macrosignature the observed minimum does lie nearest the most recent minimum- $L$ position. The relatively larger displacements at $\mathrm{MeV}$ energies in the CRS profiles are correlated with the effect of stronger sweeping at higher energies. Similar effects are also evident in Mauk et al.'s Figure 9, where the outbound proton profiles show increasingly large displacements up to $1.0 R_{U}$ in the higher-

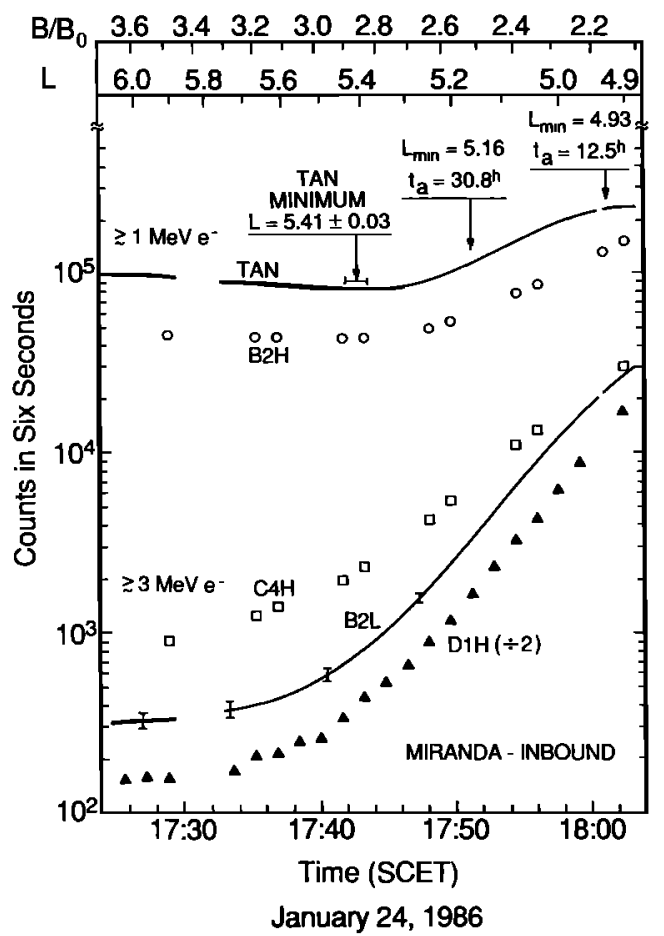

Fig. 10. Expanded time (SCET) profile from TAN for inbound macrosignature of Miranda, with 6-s counts shown from B2L rate ( $\geq 2.7-\mathrm{MeV}$ electrons) and $96-\mathrm{s}$ counts from B2H ( $\geq 1$.1-MeV electrons), $\mathrm{C} 4 \mathrm{H}$ ( $\geq 3.1-\mathrm{MeV}$ electrons), and $\mathrm{D} 1 \mathrm{H}(\geq 3.0-\mathrm{MeV}$ electrons). Minimum intensity time is delimited only for $\geq 1.1-\mathrm{MeV}$ electrons, since no local minimum is evident at higher energies. Other data are described in caption of Figure 6.

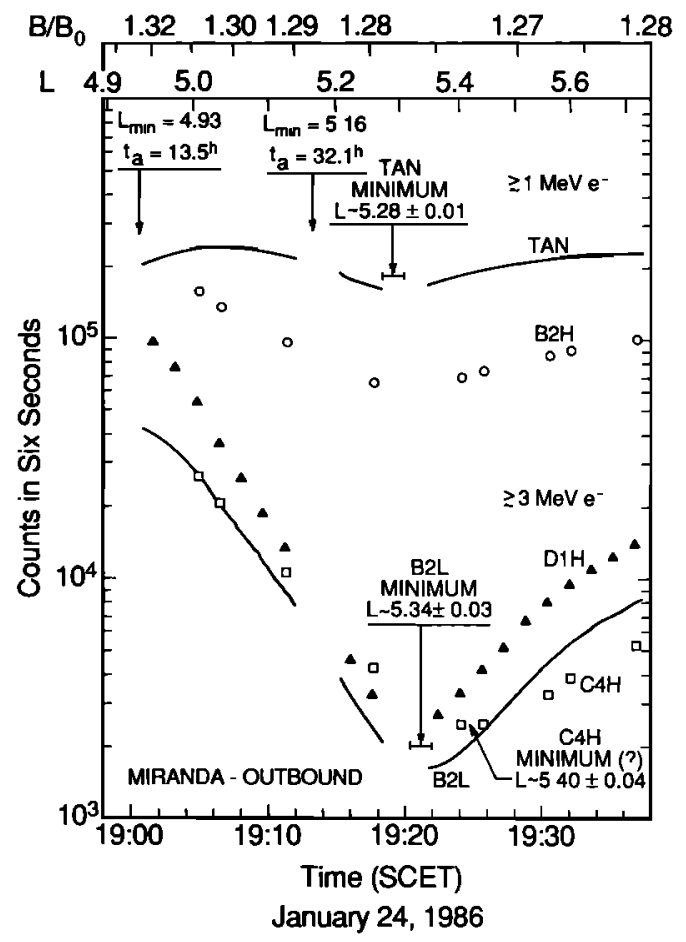

Fig. 11. Expanded time (SCET) profile for outbound macrosignature of Miranda. Refer to caption of Figure 10.

energy channels, but these are probably due to stronger dependence of sweeping on gyroradius for protons than for electrons [Paonessa and Cheng, 1987].

Because of these energy dependences, it is not possible to attribute the observed displacements to errors in the $Q_{3}$ magnetic field model. On the other hand, the displacement effect prevents more precise tests of field models with respect to locations of signature minima than that already discussed by Stone et al. [1986]. These locations were sensitive mainly to the direction and degree of tilt for the magnetic dipole axis and did not place significant constraints on higher-order, quadrupole terms in the $\mathrm{Q}_{3}$ model [Acuña et al., 1988]. A more stringent test of consistency with $\mathrm{Q}_{3}$ is provided by the location of the electron intensity maximum near closest approach in $L$ to Uranus (see section 2 ).

\section{Pitch Angle Anisotropy}

\section{Measurement Approach}

The inbound-outbound differences in the radial profiles of the macrosignatures can be used to estimate local indices ( $n$ ) for pancake-type distributions (see section 2), but the estimated values depend on assumptions about the directionality of the detector response. In general, the true anisotropies fall in the range between those estimated for a detector measuring the omnidirectional flux $J(L, B)$ and for a highly directional one measuring $j(L, B, \alpha)$. The response of a real detector involves an integration of the incident electron distributions over a finite range of angles $\theta$ and gives an observed integral intensity $J(L, B, \alpha) \sim g(\alpha, n) / B^{n}$, where $g(\alpha, n)$ is the average fraction of the omnidirectional intensity measured for a given pointing direction and anisotropy index. The calculation of $g(\alpha, n)$ requires an iterative approach since the value of $n$ is determined by the measure- 
ments. In order to allow nonintegral values of $n$ we have chosen a numerical method for evaluation of flux integrals for each stage of the iteration.

For dead-time-corrected (see the appendix) D1L event rates $R_{1}$ and $R_{2}$, respectively measured inbound and outbound, the estimated anisotropy index for a given drift shell is $n=\log \left(R_{1} / R_{2}\right) / \log \left(x_{1} / x_{2}\right)$ where $x \propto B^{-1}$ is applicable for an omnidirectional detector, $x \propto \sin ^{2} \alpha / B$ for a directional one, and $x \propto g\left(\alpha, n_{0}\right) / B$ for wide-angle response. For the latter we use the omnidirectional value of $n$ as the first estimate $\left(n_{0}\right)$, perform Monte Carlo integration to compute $g\left(\alpha_{1}, n_{0}\right)$ and $g\left(\alpha_{2}, n_{0}\right)$, and then compute a new value of $n$. Stable values of $n$ result after one or two iterations of this procedure.

The true response of the $\geq 1.1-\mathrm{MeV}$ (D1L) counting rate can characterized by a simple model due to the monotonic variation of the electron incidence threshold as a function of axial angle. The threshold has a minimal value of $1.0 \mathrm{MeV}$ on axis and increases slowly to $1.1 \mathrm{MeV}$ at $\theta \sim 40^{\circ}-50^{\circ}$, increasing rapidly thereafter to $1.6 \mathrm{MeV}$ at $60^{\circ}-70^{\circ}$ (A. C. Cummings, unpublished data, 1986). For these thresholds, and with typical energy spectra for $\mathrm{MeV}$ electrons [Stone et al., 1986], the integral energy response is an order of magnitude higher above $1.0 \mathrm{MeV}$ than above $1.6 \mathrm{MeV}$. A crude approximation to the D1L response is a uniform acceptance cone bounded by $\theta_{\max }=\cos ^{-1}(1-G / 2 \pi A) \sim$ $53^{\circ}$, where $G \sim 11 \mathrm{~cm}^{2} \mathrm{sr}$ is the bow tie geometric factor from Table Al and $A \sim 4.5 \mathrm{~cm}^{2}$ is the sensitive area of detector D1. Note that an ideal, isotropic detector (i.e., a sphere) with cross-sectional area $A$ has a total geometric factor of $4 \pi A$ and $\theta_{\max }=180^{\circ}$. Although the D1 detector actually has a flat annular geometry, which gives half as much geometric factor as a sphere for the same value of $A$ and an equivalent maximum acceptance angle of $62^{\circ}$, there is no attempt here to include the angular scattering of electrons in shielding, so there is little to be gained by more precise modeling of the detector geometry. The above model allows us to test the directional response component which is generally overwhelmed by the large latitudinal excursions of Voyager 2 in magnetic coordinates.

\section{Anisotropy Indices}

The middle panel in Figure 12 shows radial profiles of anisotropy indices calculated from inbound and outbound profiles of the D1L counting rate for the three response models. The corresponding profiles for the local pitch angle (OTD values used due to negligible differences from those of $\mathrm{Q}_{3}$; see Figure 3) of the TET boresight and for the spacecraft position in $B / B_{0}$ are shown in the top and bottom panels, respectively. As predicted, the values of $n$ for ideal responses delimit the wide-angle values from the Monte Carlo integrations. The convergence of all indices to a value near 0.25 in the Ariel macrosignature reflects the large variation in $B / B_{0}$ inbound and the nearly equal values of $\alpha_{1}$ and $\alpha_{2}$. The effect of pointing direction on the wide-angle response is potentially strongest for the anisotropy profiles in the Miranda macrosignature where $\alpha$ decreases to $30^{\circ}$ inbound and is nearly constant at $60^{\circ}$ outbound.

The striking features in all three macrosignature regions are the large dips in anisotropy toward more isotropic values, the effect being largest in the Ariel region where the measurements were made with the largest inbound-

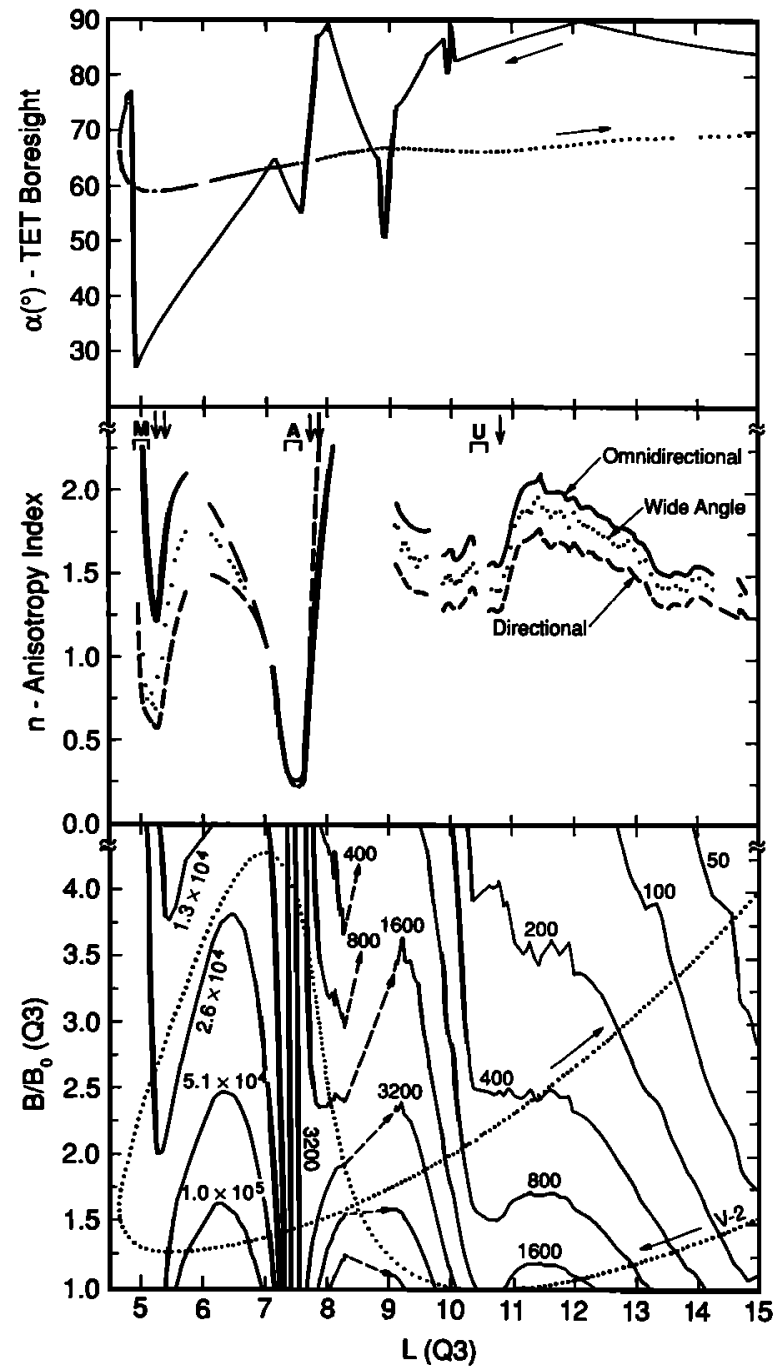

Fig. 12. (Top) Boresight direction of the electron telescope (TET) in local pitch angle $\alpha$ from the OTD field model and as a function of the local drift shell $L$ from $Q_{3}$. Inbound and outbound profiles marked by solid and dotted curves, respectively. The jagged appearance of the inbound profile is due in part to roll maneuvers which are noticeably absent outbound. (Middle) Calculated values of the anisotropy index $n$ for three models of D1L $(\geq 1.1-\mathrm{MeV}$ electrons) response: omnidirectional (solid curve), wide angle (dots), and directional (dashed curve). The excluded interval at $L \sim$ 8.3-9.1 is near the spacecraft trajectory crossover in $B / B_{0}$ where $n$ cannot be calculated accurately. The radial positions for minimum- $L$ values of satellites $(\mathrm{M}, \mathrm{A}, \mathrm{U})$ and electron intensity minima (arrows for inbound and outbound) are marked for comparison with observed dips in the anisotropy profiles. (Bottom) Isointensity contours with respect to $B / B_{0}$ and $L$ coordinates from $Q_{3}$. Each contour is mirror symmetric with respect to the minimum- $B$ equator and corresponds to a constant omnidirectional intensity in units of dead-time-corrected (see the appendix) counting rates for D1L. The wide-angle values of $\boldsymbol{n}$ are used for extrapolation from intensities measured along the Voyager 2 trajectory, which is also shown in the same coordinates. The counting rate increment between neighboring contours is a factor of 2 . Note that the contours are discontinuous in the vicinity of the crossover interval since no information on $n$ is available there.

outbound difference in $B / B_{0}$. The Ariel dip is centered within the minimum- $L$ region for that satellite and is therefore well inward from the drift shells of the measured intensity minima in the Ariel macrosignature. The anisotropy dip in the Miranda region is centered at a more 
intermediate position near the outbound intensity minimum at $L \sim 5.28$. We recall from the discussion of Figure 5 that the most isotropic point in the Miranda macrosignature profiles for $\geq 3$.1-Mev electrons occurs near $L \sim 5.4$ and not in the minimum- $L$ region. Thus the anisotropy profiles reflect to some degree the energy dependence found in the intensity minima for the Miranda region. In the Umbriel macrosignature the minimum values of $n, \sim 1.3-1.4$, lie in a broader range at $L \sim 10.0-10.8$, the inbound intensity minimum being in the vicinity of a local minimum in $n$. The small rise in $n$ within this region is associated with the outbound transition feature at $L \sim 10.34$.

\section{Intensity Contours}

The contributions of anisotropy effects to the observed macrosignatures are graphically demonstrated in the bottom panel of Figure 12, where we show isointensity contours and their relationship to the Voyager trajectory in magnetic coordinates. For given drift shells the positions in $B / B_{0}$ of contours at constant, omnidirectional intensity are calculated using the relation $B=B_{1}\left(R_{1} / R\right)^{1 / n}$, where $R$ is the contour intensity in D1L counting rate units, $R_{1}$ is the measured intensity inbound or outbound, $B_{1}$ is the field magnitude at the measurement position, and $n$ is our best estimate (i.e., the wide-angle response value) of the local anisotropy index. Note that small values of $n$ result in more nearly vertical contours (i.e., intensity is less dependent on $B / B_{0}$ ), while large values produce more nearly horizontal ones (i.e., radial intensity variations are less significant). The more nearly vertical contours cluster in the minimum- $L$ regions where observed intensities rise rapidly inward, while intensity minima occur in more anisotropic regions associated with horizontal rollovers of the contours outward from the minimum- $L$ regions.

CRS should observe local minima or maxima in intensity whenever the spacecraft trajectory crosses any contour twice in a macrosignature region, and the radial locations of these minima would be determined by the contour configurations in $L$ and $B / B_{0}$. On this basis the observations should show intensity minima for all macrosignatures, except Umbriel outbound where only single contour crossings are evident for the latter in Figure 12. As is clearly evident in the Ariel region, the more nearly vertical (i.e., isotropic) configurations of contours crossed nearer the minimum- $B$ equator are associated with smaller displacements of intensity minima from the minimum- $L$ regions, while larger displacements further away from the equator appear in conjunction with increasing anisotropy. Since larger local anisotropies are associated with relatively lower omnidirectional fluxes at higher latitudes, the intensity minima would naturally move radially outward in the direction of increasing anisotropies as the spacecraft latitude increased.

The observed depth of an intensity minimum can also be affected by the direction of the spacecraft trajectory, an example being the difference between the rather flat minimum region of the inbound Miranda signature for $\geq 1.1-\mathrm{MeV}$ electrons in comparison to the considerably greater depth outbound. This difference arose in the observations from nearly parallel movement inbound along contours at $L \sim$ 5.4-6.0 and the nearly perpendicular movement across the corresponding contours outbound. The absence of an inbound minimum for higher-energy electrons ( $\geq 3 \mathrm{MeV}$ ) in the Miranda region may be explained in a similar manner.

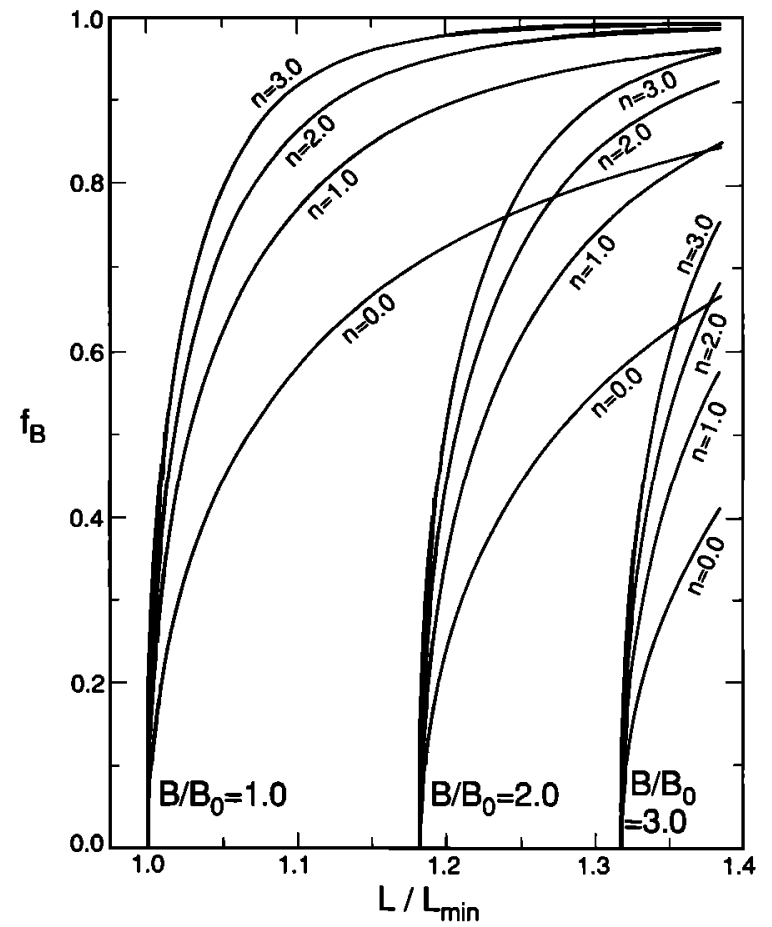

Fig. 13. Fractions of electrons observed at constant $B / B_{0}$ which mirror below the satellite orbit. These fractions are calculated as functions of $L / L_{\min }$ for different values of $B / B_{0}$ and anisotropy index $n$ of a pitch angle distribution $j(\alpha) \propto \sin ^{2 n} \alpha$.

\section{Effects on Satellite Sweeping}

Satellite absorption of the omnidirectional electron flux varies with $L \geq L_{\min }$ and $B / B_{0}$ along a satellite orbit [e.g., Paonessa and Cheng, 1987] and may have observable signatures in pitch angle distributions of affected electrons. A principal parameter is the local fraction $\left(1-f_{B}\right)$ of observable electrons which mirror above the local $B / B_{0}$ position of the absorbing satellite. These fractions are determined by the local pitch angle anisotropies. The residual fractions $f_{B}$, corresponding to those electrons mirroring below the local satellite latitude, are shown in Figure 13 for different constant values of $n$ and $B / B_{0}$ as a function of the satelliteindependent, radial parameter $L / L_{\min }$. Since the calculated curves are used only for illustrative purposes, the satellite coordinates in $B$ and $L$ are taken from the OTD field model.

The macrosignatures would be affected differently for observations at low and high magnetic latitudes by the orbital geometry. Near the magnetic equator, $f_{B}$ increases rapidly with $L / L_{\min }$ and should strongly affect the macrosignature profile. At higher magnetic latitudes all electrons mirror above the satellite orbit over increasingly wide regions, wherein $f_{B} \sim 1$ is constant. The effect of more isotropic distributions is also to increase the radial zone of potentially strong absorption and weaken the $f_{B}$ dependence. However, the observed radial widths of the macrosignatures show only weak variations with latitude, and the effects of electron sources or diffusion may be more important.

\section{Electron Sources AND Diffusion}

The observations of finite electron intensities in spatial regions swept by satellites provide clear indications of 
significant effects by radial transport processes and/or distributed, in situ electron sources. If sweeping losses were not replenished, CRS should have found negligible electron intensities as Voyager 2 passed latitudinally above the satellite orbits, where the entire electron population would eventually be absorbed in the absence of diffusion. Further indications of electron replenishment are provided by the absence of recognizable, localized absorption features near the minimum- $L$ positions of satellites, where maximal sweeping [Paonessa and Cheng, 1987] would otherwise totally deplete electrons at all pitch angles, and by the outward displacements of observed intensity minima with respect to these positions, One might expect that intensity minima for all electron energies should also be found near minimum $L$. Inconsistency of this expectation with the observations at $\mathrm{MeV}$ energies suggests that replenishment, probably driven in part by diffusion, occurs in the sweeping regions.

\section{Sweeping Time Scales}

The primary factor characterizing satellite sweeping of electrons mirroring near or above the satellite orbit is the time scale $T_{S S}$ for absorption of all such electrons within a given drift shell in the absence of radial transport. In the limit that the satellite radius $r_{s}$ is much larger than the electron gyroradius $r_{g}$, so "leapfrogging" effects can be ignored, this time scale is approximately $2 \pi T_{S} / N \omega_{r d} \Delta t . T_{S}$ is the synodic orbital period of the satellite in the corotation frame of the planetary magnetic field, $\omega_{r d}$ is the longitudinal drift frequency of the electrons relative to the Keplerian motion of the satellite in the corotation frame, $\Delta t$ is the time interval of direct contact between the local drift shell and the satellite during each single sweeping episode, and $N$ is the number of such episodes (typically four) at the same $L$ value per synodic orbit. Expressions for $\omega_{r d}$ and $\Delta t$ are given by Paonessa and Cheng [1987], Cheng et al. [1987], and Cooper [1990], the latter giving the radial dependence of $\Delta t$ at $L / L_{\text {min }} \leq 1.01$ with maximal values near $L=L_{\min }+r_{\text {eff }}$. The effective radius $r_{\text {eff }}$ for absorption is the sum of the satellite's geometric radius $r_{s}$ and the electron gyroradius $r_{g}$, the latter defining the dimension of the local electron flux tube. At $L / L_{\min }>1.01$ we use the Paonessa and Cheng approximation for $\Delta t$.

The radial dependence of the calculated sweeping times $T_{S S}$ is shown in Figure 14 for selected energies of electrons mirroring at the latitude of the satellite orbit on the local drift shell. Although the 22-keV profile for Umbriel shows an exceptionally divergent radial form as it approaches the longitudinal drift resonance limit (i.e., $\omega_{r d}$ equals satellite angular velocity $\Omega_{m}$ in corotating magnetic frame) in the 20 to $40-\mathrm{keV}$ range [Cooper, 1990] at larger $L$ values, the other profiles are dominated by the radial dependence of $\Delta t$ and show the strongest absorption where expected. For protons and higher-energy electrons the sharp spatial structure near minimum $L$ would be smoothed by leapfrogging effects.

The satellite signatures for LECP and CRS electron profiles in Figure 4 provide a measure of the most relevant time scales for satellite sweeping in the macrosignatures. The LECP profile for 112- to $183-\mathrm{keV}$ electrons shows minimal absorption features and corresponds to $T_{S S} \geq 10^{3}$ hours, the drop in intensity near 1630 SCET being mainly due to the spacecraft's large latitudinal excursion near that time. The first significant signs of absorption appear at $480-853 \mathrm{keV}$

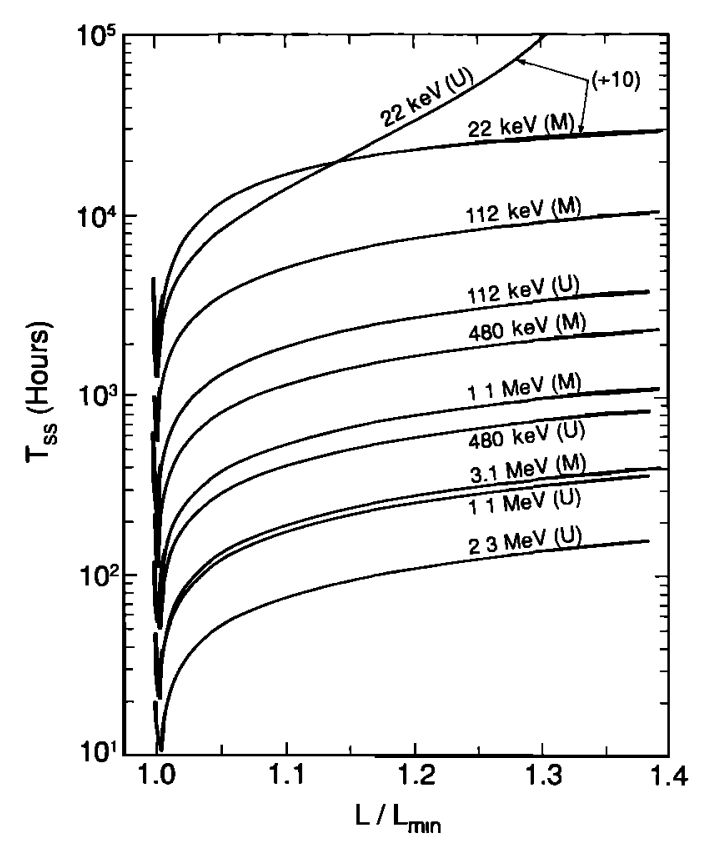

Fig. 14. Calculated sweeping times $T_{S S}$ along orbit of satellite as function of $L / L_{\min }$ for $\alpha=90^{\circ}$ at various electron energies for Miranda (M) and Umbriel (U).

where $T_{S S} \sim 10^{2}$ hours in the intensity minima near minimum $L$. Although the signatures for higher-energy electrons show deeper intensity minima, the displaced positions of these minima are located such that the local sweeping times are still about $10^{2}$ hours. The lack of observed features from sweeping on shorter time scales near minimum $L$ for the $\mathrm{MeV}$ electrons indicates that diffusion and/or other sources must compensate for rapid losses in the minimum- $L$ region. The absence of a "microsignature" in the outbound minimum- $L$ region of Umbriel has provided our best test for diffusive replenishment on small spatial scales and can be used to set limits on diffusion parameters in conjunction with calculations of sweeping times, as discussed further on in this section.

The relatively short time scales for electron replenishment in the macrosignatures rule out the extremely weak source [e.g., Blake et al., 1983; Cooper, 1983] of energetic electrons from cosmic ray albedo neutron decay (CRAND) as a probable source. The CRAND source strength for electrons and protons would be several orders of magnitude lower from cosmic ray interactions in the thin rings and the hydrogen-helium atmosphere as compared to the source from interactions with Saturn's main rings. At Saturn, trapping times of the order of 10 years are required for buildup of CRAND protons to the observed intensities, whereas trapped particles in the Uranian magnetosphere may be removed on far shorter time scales by satellite sweeping in the affected regions of the magnetosphere. The high intensities of sub-MeV electron fluxes found by LECP [Krimigis et al., 1986; Mauk et al., 1987] cannot be accounted for by beta decay except perhaps at energies near $20-40 \mathrm{keV}$ where $T_{S S}$ theoretically has very large values due to longitudinal drift resonance. Even in this case, the resonant electron lifetimes and the electron sources would be dominated by radial transport, so the CRAND source would have negligible effect, as confirmed by the lack of resonance structure in the 
LECP electron spectra at these energies [Mauk et al., 1987]. At higher electron energies the Uranian CRAND source drops rapidly above the $0.8 \mathrm{-MeV}$ beta endpoint energy for decay in the neutron rest frame. As calculated by Nakada [1963] and others, less than $10 \%$ of beta electrons are emitted at energies above $1 \mathrm{MeV}$ in the laboratory frame for typical source neutron energies of the order of $100 \mathrm{MeV}$. Only a very few electrons, emitted in the forward direction, will approach the 1.1-MeV energy threshold for parent neutrons above $26 \mathrm{MeV}$. The source neutron flux above $600 \mathrm{MeV}$, required to produce more than half the electrons above threshold, is still lower by at least another order of magnitude as compared to the 100-MeV neutron flux [e.g., Cooper, 1983].

\section{Evidence for Distributed Sources}

Analysis of phase space densities is required to determine if the source required for electron replenishment in the macrosignatures can be accounted for by inward diffusion and acceleration alone, perhaps complemented by pitch angle scattering, or if other sources, such as magnetospheric recirculation, make additional contributions. Monotonically increasing radial profiles of phase space density at increasing $L$ indicate inward diffusion, while local minima in macrosignatures point to additional sources. A positive overall radial gradient is evident in the density profiles of $\mathrm{MeV}$ electrons [Stone et al., 1986], but the profiles within macrosignatures require closer study. The report of local density minima in most macrosignatures at sub-Mev energies [Cheng et al., 1987], and the relatively deeper intensity minima found at $\mathrm{MeV}$ energies, suggest that the higher-energy measurements discussed here should provide critical tests of electron source origin.

For a phase space density $f(M, K, L)$, defined in terms of the three adiabatic trapping invariants [e.g., Schulz and Lanzerotti, 1974], the relation to differential flux $j(L, E, B$, $\alpha)$ is $f=j / p^{2}$, where an electron of mass $m$, constant first invariant $M=p^{2} \sin ^{2} \alpha / 2 m B$, and constant second invariant $K=\mathrm{J}^{2} / 8 m M$ varies in energy and local pitch angle during diffusive violation of the third invariant, $L$. The J parameter is the path integral $\oint_{p} \cos \alpha d s$ of the momentum component parallel to the local field line of differential length $d s$ between the conjugate mirror points. For equatorially mirroring electrons $(K=\mathrm{J}=0)$ the product $p^{2} L^{3}$ is constant, so such particles are accelerated during inward diffusion. In order to simplify this preliminary analysis, we assume that electrons of constant $M$ and $K>0$ move along radial paths at constant values of $B / B_{0}$, a reasonably valid approximation for local regions with radial dimensions of individual macrosignatures.

The sign of the radial gradient for local phase space density can be inferred from integral counting rate data, provided (1) that anisotropy data are available to extrapolate the measured omnidirectional intensities to arbitrary latitudinal positions on the local drift shell and (2) that a power law form $j(E) \propto E^{-\gamma}$ approximates the differential energy distribution with no significant radial variation in $\gamma$. Following the approach of McKibben and Simpson [1980], we write the $L$ dependence of the phase space density as $f(M, L) \propto$ $A(L, B) L^{3+3 \gamma / 2}$ for relativistic electrons, where $A(L, B)$ is the local integral intensity above a fixed energy threshold (e.g., $1.1 \mathrm{MeV})$. If $A(L, B)$ increases with $L$ more rapidly

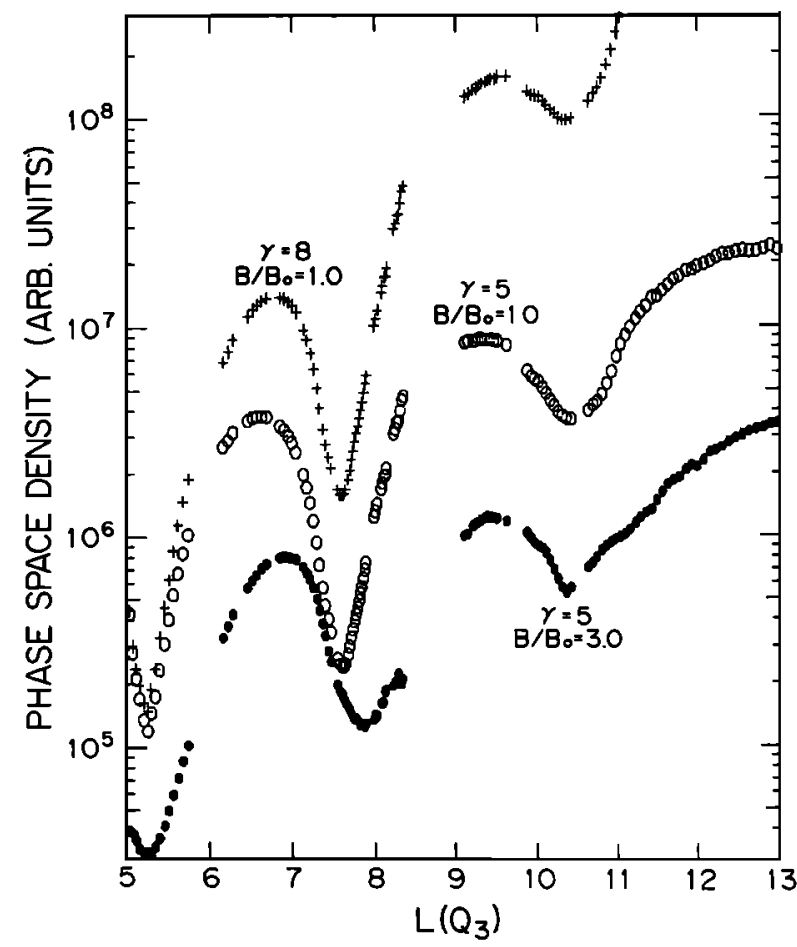

Fig. 15. Phase space density profiles versus $L\left(Q_{3}\right)$ for indicated values of the differential power law index $\gamma$ and for extrapolations of the measured intensities to different values of $B / B_{0}$. For constant values of the first adiabatic invariant $M$, the calculated phase space densities are proportional to $C(L)\left(B_{1} / B\right)^{n} L^{3+3} \gamma^{\prime 2}$ for counting rate $C(L)$ measured at field magnitude $B_{1}$ and extrapolated to $B$ with the omnidirectional values of $n$ from the middle panel of Figure 12. The selected values of $M$ are arbitrary for $L$-dependent energies above $1.1 \mathrm{MeV}$ at constant $M$.

than $L^{-3-3 \gamma / 2}, \partial f / \partial L$ is positive and the local diffusion is inward; otherwise, outward diffusion is inferred.

A key condition for the validity of the above approach is that a pancake-type distribution meaningfully represents the local pitch angle distribution. This allows the radial variation of the omnidirectional flux to be separated from latitudinal variations with respect to $B / B_{0}$ as in $J\left(L, B / B_{0}\right) \propto J_{0}(L)\left(B_{0} /\right.$ $B)^{n}$. This condition would not hold in the case of pitch angle scattering, which would also violate the second invariant, and such scattering should therefore be regarded as a potential "local source" in the event that local density minima are found.

Figure 15 shows the result of computing phase space densities with the McKibben and Simpson approach for the indicated values of $\gamma$ and $B / B_{0}$. The range $\gamma \sim 5-8$ covers the spectra above $1.1 \mathrm{MeV}$ and is based on interpretation of integral electron intensities above 1.1 and $3.1 \mathrm{MeV}$ [Stone et al., 1986] and pulse height analysis of energy deposits in the D1 detector of TET [Selesnick and Stone, 1991; J. F. Cooper, unpublished data, 1986]. The measured spectra do not show strong spectral variations in the 1- to $2-\mathrm{MeV}$ range which dominates the D1 counting rate, although it is evident from the profiles shown in Figure 4 and elsewhere that strong variations occur near and above $3 \mathrm{MeV}$ in the Miranda sweeping region. For the present analysis we believe it is sufficient to assume a constant spectral index; the work of Selesnick and Stone will address this point further. The omnidirectional fluxes at $B / B_{0}=1.0$ and 3.0 are extrapo- 
lated using the omnidirectional values of the pancake index $(n)$ in Figure 12. The extrapolation is done with respect to dead-time-corrected (see the appendix) D1L event rates measured along the Voyager trajectory in $L$ and $B / B_{0}$ coordinates. The missing data points at $L=8.4-9.0$ arise from errors in calculating $n$ near the inbound-outbound latitudinal crossover, while other breaks in the profiles are due to data gaps.

Independent of the assumed spectral index or latitudinal location, there are clearly deep density minima in the macrosignatures of Miranda, Ariel, and Umbriel. These features cannot be removed by reasonable radial variations of the spectral index within the limits of the measurements at 1-2 $\mathrm{MeV}$. The radial dimensions of the density macrosignatures are comparable to those of the intensity macrosignatures, although the anisotropy structure does produce some quantitative differences in the shape and depth of the profiles. The Ariel density profile at high latitude has an obvious outward displacement with respect to the local minima of the equatorial profiles, which show no significant spectral dependence. The overall decline in density inward from $L=13$ suggests a strong, if not dominant, role by inward diffusion. The local density minima may then indicate additional contributions by internal sources and/or the nonadiabatic effects of pitch angle scattering.

\section{Diffusion Coefficients}

The transport of trapped radiation at constant first $(M)$ and second $(K)$ invariant is represented by the differential equation [Schulz and Lanzerotti, 1974]

$$
\frac{\partial f}{\partial t}=L^{2} \frac{\partial}{\partial L}\left(\frac{D_{L L}}{L^{2}} \frac{\partial f}{\partial L}\right)_{M, K}+\mathrm{S}_{M, K}-\mathrm{L}_{M, K}
$$

in which the radial diffusion coefficient is often parameterized as $D_{L L} \propto L^{m}$. The assumption of time independence (i.e., long-term averaging) gives $\partial f / \partial t=0$, while the loss term $\mathrm{L} \sim f / T_{S S}$ is determined from the measurements and satellite sweeping theory. For inward diffusion the volume source term $S$ is zero, but distributed sources would give nonzero values.

The most rigorous approach toward deriving values of $D_{L L}$ is to explicitly define the source and loss terms (e.g., via satellite sweeping theory), and their dependences on $M, K$, and $L$, solve equation (1) for $f$ in terms of these parameters, and determine values of constant coefficients in terms of least squares fits to the measured phase space density profiles. In the simplest case, where time dependence, pitch angle scattering, and local sources are neglected, this approach gives well-determined values of the diffusion coefficient and its radial dependence as found in the work of Cheng et al. [1987] and Hood [1989], who analyzed the LECP phase space density data. However, since this case does not allow for effects of local sources, the fits are best for data without significant local minima in macrosignatures. This limitation confines the validity of the analytic approach to the lowest LECP particle energies which show minimal, if any, local minima in intensity or phase space density profiles. At higher energies one must explicitly include finite source terms to obtain meaningful fits to the CRS macrosignatures (R. S. Selesnick and E. C. Stone, unpublished manuscript, 1990). Since we can only guess at the functional dependence on the independent variables of the source term, in that we do not know the source a priori, the parameters fits for $D_{L L}$ and $S$ will retain large uncertainties until the sources are better understood. In the present analysis we instead utilize an empirical method for estimating $D_{L L}$ which is based on dimensional arguments and gross physical parameters (e.g., radial width and average sweeping rate) for the observed macrosignatures. The empirical values of $D_{L L}$ are then compared with those from the two LECP analyses cited above and found to be in reasonable agreement.

The published works on electron sweeping by Io in the Jovian magnetosphere provide comparative tests of different empirical methods for estimating $D_{L L}$. For an Io sweeping region of radial dimension $\Delta L, M o g r o-C a m p e r o$ and Fillius [1976] used a simple coefficient of the form $D_{L L} \sim(\Delta L)^{2}$ / $4 T_{D}$, where a time scale $T_{D}$ is required for an electron to diffuse across this region. In equilibrium this time scale is nominally equal to that required for sweeping to reduce the ambient electron intensity (i.e., in the absence of sweeping) to the observed minimum level within the satellite signature. The above authors also used a second method incorporating information on measured local gradients in phase space density. The two methods yielded diffusion coefficients in the range of $3 \times 10^{-8}$ to $4 \times 10^{-7} R_{J}^{2} / \mathrm{s}$, the lower value being calculated by the first method. Thomsen et al. [1977] performed more elaborate calculations, solving for lossy diffusion within the Io sweeping region and lossless diffusion outside that region, and finding an Io value of $4 \times 10^{-7} R_{J}^{2} / \mathrm{s}$, in reasonable agreement with that from the second method of Mogro-Campero and Fillius.

Since all the above values for $D_{L L}$ differ only by an order of magnitude for the case of electrons at Io's orbit, we postulate that an intermediate value, $D_{L L} \sim(\Delta L)^{2} / T_{D}$, provides a reasonable estimate which can be applied to our electron macrosignatures at Uranus. We define the characteristic radial dimensions of the observed macrosignatures at Uranus to be twice the displacements of the intensity minima from the time-averaged minimum- $L$ values of the parent satellites. Although these dimensions, listed in Table 2, may not be the same as those more correctly calculated from phase space densities, it is obvious from the radial intensity profiles that widths of regions visibly affected by sweeping are roughly approximated by the listed dimensions, which differ by less than a factor of 2 between Miranda and Umbriel. Using the relatively broad dimensions of the diffusive profile, as recommended by Thomsen et al. [1977] in regard to Io, ensures that the estimated values of $D_{L L}$ are minimally affected by time-dependent effects of episodic sweeping.

We further assume that the average sweeping time $T_{S S}$ at the intensity minimum, also listed in Table 2, roughly approximates $T_{D}$ within an order of magnitude. Since these sweeping times are $10^{2}-10^{3}$ hours, they are appropriate to the approach of Thomsen et al. for long-term averaging over many individual sweeping episodes. Since no signs of local sweeping appear near minimum $L$ in CRS macrosignatures with local intensity and density minima, it would not be valid to use the minimum- $L$ sweeping rate, as done by Cheng et al. [1987] for the low-energy electron signatures from LECP, to model losses characteristic of the CRS macrosignatures.

Our estimated values for $D_{L L}$ in Table 2 lie in the range $10^{-7}-10^{-6} R_{U}^{2} / \mathrm{s}$ for the observed macrosignatures and agree within a factor of 2 for different $B / B_{0}$ on the same $L$ shell. 
TABLE 2. Estimated Diffusion Coefficients From Satellite Signatures

\begin{tabular}{cccccccc}
\hline Satellite $^{a}$ & IB/OB $^{b}$ & MA/MI & $L$ & $B / B_{0}{ }^{d}$ & $\Delta L$ & $T_{S S}$ & $D_{L L}, R_{U}^{2} / \mathbf{s}$ \\
\hline M & IB & MA & 5.41 & 2.86 & 0.65 & 401 hours & $2.9 \times 10^{-7}$ \\
M & OB & MA & 5.28 & 1.28 & 0.39 & 282 hours & $1.5 \times 10^{-7}$ \\
A & IB & MA & 7.84 & 3.09 & 0.75 & 144 hours & $1.1 \times 10^{-6}$ \\
A & OB & MA & 7.70 & 1.50 & 0.47 & 105 hours & $5.8 \times 10^{-7}$ \\
U & IB & MA & 10.74 & 1.00 & 0.70 & 81 hours & $1.7 \times 10^{-6}$ \\
U & OB & MI & 10.286 & 2.10 & 0.04 & 53 min & $>5.0 \times 10^{-7}$ \\
\hline
\end{tabular}

${ }^{a}$ Miranda (M), Ariel (A), or Umbriel (U).

${ }^{b}$ Inbound (IB) or outbound (OB) signature.

${ }^{c}$ Macrosignature (MA) or microsignature (MI).

${ }^{d}$ Values for Voyager 2 only.

Increasing radial displacements at higher $B / B_{0}$ give correspondingly larger empirical values of $D_{L L}$, although these may be artifacts of our estimation method. In comparison, Cheng et al. [1987] found $D_{L L} \sim(5-9) \times 10^{-7} R_{U}^{2} / \mathrm{s}$ from the LECP measurements of phase space densities for sub-MeV electrons in the Ariel macrosignature. The more recent analysis by $H o o d$ [1989] finds $\sim 6 \times 10^{-7} R_{U}^{2} / \mathrm{s}$ for electrons at constant $M=50 \mathrm{MeV} /$ gauss near $L=7.7$, which corresponds to the lowest LECP electron response range of 22-35 keV. Since these LECP values are close to those in Table 2 for Ariel within uncertainties at the order of magnitude level, we do not resolve any significant energy dependence in the $D_{L L}$ values derived from low- and high-energy electron measurements. This level of agreement is very surprising in view of the completely different physical assumptions (i.e., radially monotonic profile versus local minima) and needs to be confirmed by more quantitative work.

The predicted (but undetected) outbound encounter with an Umbriel drift shadow (see section 4) provides a lower limit from microsignature analysis which is consistent with the above values. As shown previously in Figure 7, the potentially observable shadow in 1.1- to $1.5-\mathrm{MeV}$ electrons would have been traversed by Voyager 2 at $L=10.275$ $10.316\left(\mathrm{Q}_{3}\right)$, where $\Delta L \sim 0.04$ and $t \sim 53 \mathrm{~min}$, for drift over a magnetic longitude interval of $277^{\circ}$. The initial depletion at 1.1-1.5 MeV would have been nearly total due to the equatorial location of Umbriel near minimum $L$ at 10.275 . Diffusive fill-in to a fractional level of 0.7 would give a limit $D_{L L}>5 \times 10^{-7} R_{U}^{2} / \mathrm{s}$, based on comparison to diffusive profiles calculated for microsignatures by Van Allen et al. [1980]. The slope transition of the macrosignature profile at this position may have made shallower, but broader microsignatures more difficult to detect. For fill-in levels above 0.9 the corresponding limits would be $D_{L L}>4 \times 10^{-6} R_{U}^{2} / \mathrm{s}$. In the Umbriel sweeping region the macrosignature value $D_{L L} \sim 2 \times 10^{-6} R_{U}^{2} / \mathrm{s}$ agrees at the order of magnitude level with these limits.

The similar radial widths of the Miranda, Ariel (outbound), and Umbriel macrosignatures suggest that $D_{L L}$ cannot vary strongly with $L$ if $D_{L L}$ is independent of energy and if we ignore the relatively small radial variations in $T_{s s}$. At comparable positions in $B / B_{0}$ the power law dependence of $D_{L L}$ on $L$ is $L^{m}$, where $m \sim 3.2-3.6$ is consistent with values of $D_{L L}$ in Table 2. Since $D_{L L} \sim L^{10}$ would be more characteristic of diffusion driven by externally induced fluctuations in magnetospheric fields [e.g., Schulz and Lanzerotti, 1974], the radial transport of energetic electrons at Uranus may be driven by other processes. The apparent increase in $D_{L L}$ with magnetic latitude would also be incon- sistent with diffusion driven directly by magnetic impulses, which preferentially affect particles trapped near the equator [see Schulz and Lanzerotti, 1974, Figure 26].

The inferred radial dependence is similar to that expected for diffusion driven by an ionospheric dynamo, analogous to that proposed to account for high diffusion rates in the inner magnetosphere of Jupiter [e.g., Schulz, 1979]. Hood [1989] also finds a low-order $L$ dependence for low-energy electrons and ions in the LECP macrosignatures and concludes that the ionospheric dynamo is dominant. He further notes that the random electric field variations expected from the dynamo process at Uranus would be sufficient to produce the inferred diffusion rates. Such $L$ dependence is also invoked to describe low-altitude transport in the magnetospheric recirculation model [Fujimoto and Nishida, 1990a, b], which may explain the anisotropy variations within the CRS macrosignatures.

\section{Conclusions}

The observed profiles of the macrosignatures are best interpreted as quasi-stable features in which the timeintegrated effects of electron source injection, satellite sweeping, and radial transport are in equilibrium. The lack of correlation for observed intensity minima with the most recent positions of maximal sweeping clearly shows the lack of time dependence with respect to individual sweeping episodes as each satellite crosses the minimum- $B$ equator. The presence of electron intensity minima in regions where sweeping time scales are long $\left(10^{2}-10^{3}\right.$ hours $)$ is indicative of minimal effects from individual sweeping episodes occurring at much shorter intervals. The observations of nonzero electron intensities in regions swept at any finite level by the satellites prove that the electrons are continuously replenished by some source process, which can be either direct local injection from a distributed, internal source or radial transport into the swept region from injection sites elsewhere in the magnetosphere. The local minima in phase space density profiles for sub-MeV electrons from LECP [Cheng et al., 1987], and the similar results for $\mathrm{MeV}$ electrons from our present work, are consistent with effects of distributed source injection which replenish the swept regions via inward and outward diffusion from neighboring regions where sweeping is less effective and injected electrons build up to higher intensity levels.

Radial inward diffusion and acceleration provides a wellknown source of $\mathrm{MeV}$ electrons from lower-energy reservoirs in the outer magnetosphere. Generally positive radial gradients in phase space densities of such electrons [Stone et 
al., 1986] indicate that such adiabatic diffusion processes play a significant role at Uranus. Although the $\sim L^{3}-L^{4}$ radial dependence of our macrosignature-derived values for $D_{L L}$ is inconsistent with that expected for conventional diffusion driven by magnetic or electric impulses, it is similar to that expected for diffusion driven by electric fields from the ionospheric dynamo [e.g., Schulz, 1979]. Recent work by Hood [1989] gives a similar result from modeling of phase space densities derived from LECP data for sub-MeV electrons and protons.

Since phase space density analysis indicates deviations from simple inward diffusion in regions of strong losses to satellite sweeping, additional sources from magnetospheric recirculation or other processes requiring nonadiabatic transport modes may be required. The case for recirculation is currently supported by the more isotropic electron distributions near regions of strong sweeping, where this process may provide the most effective replenishment source. The recirculation model has been recently successful in accounting for energetic electron measurements of spectra and anisotropies at Earth [Fujimoto and Nishida, 1990a] and Jupiter [Fujimoto and Nishida, 1990b] and may be generally applicable to Uranus as well. Alternatively, effects of pitch angle scattering alone [Selesnick and Stone, 1991] might supplement transport by adiabatic inward diffusion and account for the anisotropy results.

In presenting a more detailed view of time intensity profiles for $\mathrm{MeV}$ electrons, we have also compared the observed magnetic coordinates of intensity minima and maxima with predictions from the $\mathrm{Q}_{3}$ model for the planetary magnetic field. In the special case of Voyager 2's passage through its minimum $L$ during the encounter, we have found excellent agreement $(\Delta L \sim 0.01)$ between the times for predicted and measured maximum intensity, having established that maximum intensity should be found near the minimum- $L$ position. In that case we have verified that the simpler offset, tilted dipole (OTD) model does not allow accurate calculation of $L$.

On the other hand, we have also graphically demonstrated that measured positions of minimum electron intensity are close to, but not coincident with, the minimum- $L$ positions of satellites, where their sweeping rates are maximal. Electron absorption by the Uranian satellites, particularly at energies above a few hundred $\mathrm{keV}$, occurs over finite radial zones and is not localized at minimum $L$. Although the observed displacements of intensity minima from the minimum $L$ are indicative of effects from diffusion and/or distributed electron sources, these displacements are energy dependent and cannot directly constrain magnetic field models beyond the level already achieved in the preliminary CRS analysis by Stone et al. [1986].

The 1986 encounter with Uranus has given us a brief but informative "snapshot" of an energetic electron population which shows strong effects of satellite sweeping at higher energies in the unique tilted field geometry of the planetary magnetosphere. The deconvolution of various effects due to field geometry, sources, sweeping, and diffusion at Uranus will be difficult but challenging. Although much of the magnetospheric physics at Uranus is necessarily similar to that at Earth, satellite sweeping and macrosignature formation provide unique probes of magnetospheric dynamics and new insights into the general physics of planetary magnetospheres.

\section{APPENDIX: CRS INSTRUMENTATION AND RESPONSE}

The full complement of charged particle telescopes in the CRS includes two high-energy telescopes (HET-I and HETII), four low-energy telescopes (LET-A, B, C, and D), and the electron telescope (TET) [Stone et al., 1977; Stilwell et al., 1979]. Previous radiation damage or electronic malfunctions precluded multiple-coincidence analysis for HET-I and LET-B. For the Uranus encounter the configurations of all CRS telescopes were chosen to allow optimal observations over a wide range of potential trapped radiation intensities [Stone et al., 1986]. HET-I cycled every 3.2 min between high- and low-gain states for measurements of low- and high-intensity radiation environments, whereas HET-II was operated continuously in low-gain mode. The TET configuration provided continuous energy analysis of electron events in the front D1 detector, while also allowing analysis of multiple-coincidence events in the other detectors, D2D7. Events in the anticoincidence shields of HET-I and TET were excluded from analysis to prevent excessive analysis dead time in the event of high shield event rates.

The cycling and rate sampling frequencies were kept at the same level throughout the encounter, primarily because we could not anticipate the morphology of an unknown magnetosphere. The highest time resolution was provided by the TAN counting rate and by B2L (HET-II), these rates being continuously accumulated at 6-s intervals. The D1L, D1H, and other singles rates were sampled every $96 \mathrm{~s}$ in 6-s accumulation intervals, but the HET-I gain cycling produced lower sampling frequencies for some rates. Large gaps in B2H (HET-I) coverage relative to B2L resulted, for example, from 3.2-min intervals when HET-I was in low-gain mode. The steplike appearances of some consecutive 6-s counts (see Figure 6) were due to digitization error in eight-bit rate accumulators which prevent resolution of fractional changes smaller than $\mathbf{0 . 0 0 4}$.

\section{Thresholds and Geometry Factors}

Because of high electron intensities at Uranus and large accidental coincidence rates in the CRS telescopes, the CRS electron analysis was limited to counting rates and pulse height data from single detectors. The nominal threshold energies and geometric factors for electron counting in each detector are listed in Table Al and were determined with extrapolated range data [e.g., Evans, 1955] from the angledependent shielding around the detector and from the electronic discriminator threshold for energy deposits in the detector. The bow tie method [Fillius and Mcllwain, 1974; Van Allen et al, 1974] was used to minimize dependence of calculated response parameters on the energy spectra of incident electrons [Stone et al., 1986]. (Recent laboratory calibrations, utilizing a radioactive source $\left({ }^{106} \mathrm{Ru}\right)$ and a magnetic spectrometer (R. S. Selesnick and M. D. Looper, unpublished data, 1989), verify that the D1 response is reasonably well approximated by the bow tie method.) For electrons we have also tabulated the energies $E_{e w}$ for penetration of the detector windows and the energies $E_{e s}$ for penetration of the detectors. The intermediate energy range $E_{e w}-E_{e s}$ establishes the range in which all residual electron energy is deposited in the detector.

The nominal response parameters in Table Al may not characterize the true response for those detectors with 
TABLE A1. Nominal Voyager 2 CRS Detector Characteristics

\begin{tabular}{|c|c|c|c|c|c|c|c|}
\hline \multirow[b]{2}{*}{ Telescope } & \multirow[b]{2}{*}{ Detector $^{a}$} & \multirow{2}{*}{$\begin{array}{c}\text { Electronic } \\
\text { Threshold, } \\
\text { MeV }\end{array}$} & \multicolumn{4}{|c|}{ Energy Thresholds ${ }^{b}$} & \multirow{2}{*}{$\begin{array}{c}\text { G Factor } \\
A \Omega, \\
\mathrm{cm}^{2} \mathrm{sr}\end{array}$} \\
\hline & & & $\begin{array}{l}E_{\text {ew }}, \\
\mathrm{MeV}\end{array}$ & $\begin{array}{l}E_{e n}, \\
\mathrm{MeV}\end{array}$ & $\begin{array}{l}E_{e s}, \\
\mathrm{MeV}\end{array}$ & $\begin{array}{l}E_{\text {pn }}, \\
\mathrm{MeV}\end{array}$ & \\
\hline TET & D1L & 0.5 & 0.8 & 1.1 & 2.1 & 13 & 11 \\
\hline HET-I & B2H & 0.3 & 0.9 & 1.1 & 1.8 & 16 & 13 \\
\hline HET-I/II & B2L & 2.0 & 0.9 & $(2.7)^{d}$ & 1.8 & 16 & $(17)^{d}$ \\
\hline TET & D1H & 2.5 & 0.8 & $(3.0)^{d}$ & 2.1 & 14 & $(12)^{d}$ \\
\hline HET-I & $\mathrm{C} 4 \mathrm{H}$ & 1.0 & 2.3 & 3.1 & 4.9 & 28 & 21 \\
\hline HET-I & $\mathrm{C} 2 \mathrm{H}$ & 1.0 & 2.3 & 3.1 & 5.0 & 28 & 11 \\
\hline HET-I & $\mathrm{C} 3 \mathrm{H}$ & 1.0 & 5.1 & 6.0 & 7.8 & 47 & 42 \\
\hline HET-I/II & C4L & 5.3 & 2.3 & $(7.6)^{d}$ & 4.9 & 30 & $(13)^{d}$ \\
\hline HET-I/II & $\mathrm{C} 2 \mathrm{~L}$ & 5.3 & 2.3 & $(8.5)^{d}$ & 5.0 & 30 & $(13)^{d}$ \\
\hline HET-I/II & C3L & 5.3 & 5.1 & $(10.3)^{d}$ & 7.8 & 48 & $(30)^{d}$ \\
\hline
\end{tabular}

${ }^{a}$ The letters $\mathrm{L}$ and $\mathrm{H}$ denote low and high electronic thresholds, respectively, for the TET D1 detector. For the HET rates these letters denote low-gain $(\mathrm{L})$ and high-gain $(\mathrm{H})$ states corresponding to high and low electronic thresholds, respectively.

${ }^{b} E_{e n}$ and $E_{p n}$ are the nominal electron and proton energy thresholds, respectively. $E_{e w}$ to $E_{e s}$ is the range of incident energy for vertically incident electrons to stop in the detectors and be counted with unit efficiency [e.g., Lupton and Stone, 1972]. All protons at energies near $E_{p n}$ will stop in the corresponding detectors.

${ }^{c}$ Bow tie geometric factors calculated for nominal electron threshold. These do not include electron detection efficiencies.

${ }^{d}$ The nominal response parameters may not accurately represent the actual electron response if $E_{e n}>E_{e s}$ (see section 4).

sufficiently high electronic thresholds that $E_{e n}>E_{\text {es }}$. In these cases the actual detector responses would most likely be weighted toward larger incidence angles where reduced geometric factors allow longer path lengths through the detector and satisfy the triggering condition that $E_{e s} \geq E_{e n}$. The corresponding energy thresholds in these cases would depend on the external shielding configurations at the larger incidence angles. If the energy spectra of the incident electrons happen to fall off so rapidly with energy that relatively few electrons are incident at energies above the nominal threshold, the detector response could become dominated by pulse pileup from multiple electrons at energies in the range $E_{e w}$ to $E_{e s}$. Although these more complex response factors require further study, we will refer to the nominal parameters for the present work.

\section{Electron-Proton Discrimination}

The relative sensitivity of single CRS detectors to electrons and protons may be assessed in part from the data in Table A1, where we have also tabulated the proton energy thresholds computed from the same external shielding parameters as were used to calculate the nominal electron thresholds. The thresholds are generally much smaller for electrons, which would then dominate the response of each detector for incident particle spectra falling off rapidly at $\mathrm{MeV}$ energies, even if the electron and proton fluxes at the same energy were comparable. The pairs of low and high electronic thresholds for the same detectors provide the best available test for the presence of a higher-energy proton component. The increases in the electronic threshold greatly change the electron thresholds but have little effect on those for protons. For example, the D1 threshold increases from 1.1 to $3.0 \mathrm{MeV}$ for electrons but only from 13 to $14 \mathrm{MeV}$ for protons. For most other detectors the changes in proton thresholds are less than $1 \mathrm{MeV}$. This effect arises from the relatively greater fraction of incident energy lost by the protons in penetrating external shielding and from the relatively larger decrease in differential energy loss per unit path length with increasing proton energy. If the observed time profiles and counting rates from the same detector at dif- ferent electronic thresholds were comparable, a significant proton response would be clearly indicated. Otherwise, a much larger response at the lower threshold would be indicative of electrons.

There is no direct evidence for a significant high-energy proton component in the part of the Uranian magnetosphere explored by Voyager 2. Initial reports of energetic particle spectra at Uranus [Stone et al., 1986; Krimigis et al., 1986] indicate that proton fluxes are generally lower than those of electrons up to $10 \mathrm{MeV}$, the upper limit of the available measurements for finite proton fluxes. A significant highenergy component above $63 \mathrm{MeV}$ was ruled out near closest approach to the planet by Stone et al. They also defined an $L$-dependent momentum limit $7.0 / L^{2} \mathrm{GV}$ for stable adiabatic trapping of charged particles. This limit precludes significant trapped proton fluxes at $L \geq 6.6$. On the other hand, their analysis did not rule out protons in the 13- to $63-\mathrm{MeV}$ range in the Miranda sweeping and close approach regions. However, the very different counting rates registered at different energy thresholds in these regions for the same physical detectors (e.g., D1L-D1H, C4H-C4L, C2H-C2L; see Figures 10,11 , and A1) argue against significant proton fluxes in this intermediate range.

The different shapes of energetic electron and proton time profiles also allow a measure of discrimination for particle type. The relative levels of the counting rate peaks at $\mathbf{1 8 3 0}$ SCET and 2000 SCET in Figure 4 are characteristic of energetic electron response at energies above $10^{2} \mathrm{keV}$, whereas the LECP proton counting rates at $0.2-2 \mathrm{MeV}$ [Krimigis et al., 1986; Mauk et al., 1987] and the 22- to 35-keV electron profile in Figure 4 show a higher peak near 2000 SCET than at the spacecraft's minimum- $L$ value. This second peak is associated with the spacecraft's proximity to the minimum- $B$ equator as shown in Figure 2. The LECP authors noted that the inbound fluxes for protons above 0.54 MeV were 3 orders of magnitude lower than the corresponding outbound fluxes in the radial region between the minimum- $L$ positions of Miranda and Ariel. This large inboundoutbound asymmetry probably arises from the extreme anisotropy of pitch angle distributions for stably trapped 


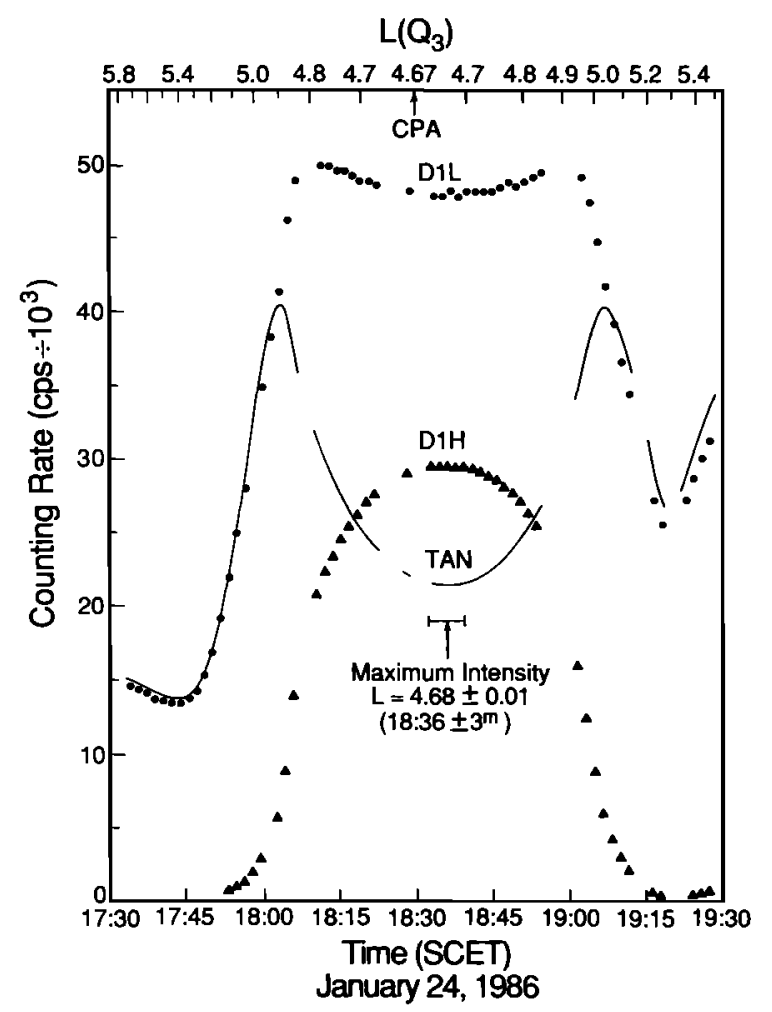

Fig. A1. Integral counting rates in the inner magnetosphere for three discriminator levels of the D1 detector in the electron telescope (TET): D1L ( $\geq 1.1-\mathrm{MeV}$ electrons), D1H $(\geq 3.0-\mathrm{MeV}$ electrons), and TAN (D1L. D1H). The TAN rates are shown at maximal resolution of $6 \mathrm{~s}$ with broken curves, the breaks indicating data gaps, while the other two rates are available in one 6-s interval every $96 \mathrm{~s}$. The vertical arrows mark the closest point of approach (CPA) in $L$ to Uranus and the approximate location of maximum electron intensity. Note that TAN reaches a local minimum near CPA due to the high veto rate from $\mathrm{D} 1 \mathrm{H}$.

protons at higher energies in this region. In comparison the dead-time-corrected counting rates for $\mathrm{MeV}$ electrons from CRS differ by no more than 1 order of magnitude inbound and outbound in the same region, so significant contributions to the nominal electron profiles from protons at much higher energies are improbable.

\section{Linearity of Counting Rates}

The highest counting rate levels were registered by CRS inside the orbit of Miranda and provided an opportunity to test the nonlinear response of the CRS counting electronics under extreme conditions. In Figure $\mathrm{A} 1$ the following counting rates are shown for the period 1730-1930 SCET from three D1 discriminators: D1L, D1H, and TAN. The first two respond to energy deposits above 0.5 and $2.5 \mathrm{MeV}$, respectively, while the third triggers pulse height analysis for D1L events with no D1H trigger (i.e., TAN events satisfy the logic condition D1L. $\overline{\mathrm{D} 1 \mathrm{H}}$ ).

The D1L rate saturates at $\approx 5 \times 10^{4}$ counts per second (cps), corresponding to a minimum retrigger time $\tau_{r} \sim 20 \mu \mathrm{s}$ for the associated electronic discriminator in CRS, which gives us a key parameter to estimate dead time corrections elsewhere. The true D1L incidence rate $R$ can then be approximated by $R \sim C /\left(1-C \tau_{r}\right)$ in terms of $C$, the observed raw counting rate. For example, the true rate near
1430 SCET at a local intensity peak would be about $25 \%$ higher than the observed rate. The lower count rates of $10^{3}$ cps near 1345 SCET and 1600 SCET would require dead time corrections of only $2 \%$. We therefore conclude that CRS counting rates near minimum levels in satellite absorption signatures are sufficiently linear to allow accurate measurements of the positions of those minima without discriminator dead time corrections. We do, however, make these corrections to facilitate latitude extrapolations of omnidirectional fluxes in the CRS anisotropy (see section 5) and phase space density (see section 6) analyses.

The TAN rate response in the close approach region is more complex, because of the anticoincidence with $\mathrm{D} 1 \mathrm{H}$ events, and shows a rollover at $4 \times 10^{4} \mathrm{cps}$ and a decline toward a local minimum of $2 \times 10^{4} \mathrm{cps}$ which occurs at 1836 ( $\pm 3 \mathrm{~min}$ ) SCET and $L=4.68$. This position is very near that of minimum $L$ for Voyager 2 , as discussed in section 2 . The maximum D1H rate and the minimum TAN rate correspond to the maximum electron fluxes which are observed within $\Delta L \sim 0.01$ of the closest point of approach in $L$ to Uranus at $L \sim 4.67$. The sum of TAN and D1H rates is nearly the saturated D1L rate, consistent with the trigger condition for TAN. The slight depression of D1L near 1830 SCET may be due to baseline shift and/or pileup effects, but the ratio of the observed D1L and TAN rates is determined linearily by the D1H anticoincidence as expected. Thus we see no evidence of significant electronic distortions which might have affected counting rates with higher energy thresholds in this region.

Acknowledgments. We thank R. E. Vogt for his contributions during the Jupiter and Saturn encounters as principal investigator for the CRS. Our present work owes a great debt to D. L. Chenette's pioneering contributions to CRS data analysis during the encounter with Uranus. We are also indebted to $M$. Acuña, J. E. P. Connerney, and $\mathrm{N}$. Ness for providing $B$ and $L$ coordinates prior to publication for Voyager 2 and the satellites from the $Q_{3}$ model for the planetary magnetic field. We thank A. C. Cummings, M. Tranh, D. Burke, and J. Weger of Caltech for technical assistance in the performance of laboratory experiments to evaluate the responses of CRS detectors to energetic electrons. The continued productivity of the CRS experiment has also been due in part to technical support provided by W. E. Althouse, T. Garrard, and R. Burrell at Caltech, N. Lal at Goddard Space Flight Center, and O. Divers at the Jet Propulsion Laboratory. The principal data analysis for this report was carried out during J. Cooper's residence as a postdoctoral fellow at Caltech under the support of NASA contracts NAS7-918 and NGR 05-002-160. J.F.C. also thanks J. P. Wefel of the Department of Physics and Astronomy at Louisiana State University for cooperation and support during completion of the final manuscript. Partial support was provided at LSU by ONR grant N-00014-90-J1466 and NASA grant NAG 2-528. Additional support was provided to J. Cooper by ST Systems Corporation (STX).

The Editor thanks L. Hood and B. Randall for their assistance in evaluating this paper.

\section{REFERENCES}

Acuña, M. H., J. E. P. Connerney, and N. F. Ness, Implications of the GSFC $Q_{3}$ model for trapped particle motion, J. Geophys. Res., 93, 5505, 1988.

Baker, D. N., J. B. Blake, L. B. Callis, R. D. Belian, T. E. Cayton, and T. A. Fritz, Relativistic electrons near geostationary orbit: Evidence for internal magnetospheric recirculation, Geophys. Res. Lett., 16, 559, 1989.

Blake, J. B., H. H. Hilton, and S. H. Margolis, On the ejection of cosmic ray secondaries into the inner Saturnian magnetosphere, 1, Protons from the CRAND process, J. Geophys. Res., 88, 803, 1983. 
Chenette, E. L., E. C. Stone, J. F. Cooper, A. C. Cummings, F. B. McDonald, J. H. Trainor, N. Lal, and R. McGuire, High energy particles: The Cosmic Ray System (CRS) experiment preliminary results from the Voyager 2 encounter with Uranus, paper presented at Spring Meeting, AGU, Baltimore, Md., May 19-22, 1986.

Cheng, A. F., S. M. Krimigis, B. H. Mauk, E. P. Keath, C. G. Maclennan, L. J. Lanzerotti, M. T. Paonessa, and T. P. Armstrong, Energetic ion and electron phase space densities in the magnetosphere of Uranus, J. Geophys. Res., 92, 15,315, 1987.

Connerney, J. E. P., M. H. Acuña, and N. F. Ness, The magnetic field of Uranus, J. Geophys. Res., 92, 15,329, 1987.

Cooper, J. F., Nuclear cascades in Saturn's rings: Cosmic ray albedo neutron decay and origins of trapped protons in the inner magnetosphere, J. Geophys. Res., 88, 3945, 1983.

Cooper, J. F., Satellite sweeping of electrons at Neptune and Uranus, Geophys. Res. Lett., 17, 1665, 1990.

Evans, R. D., The Atomic Nucleus, p. 624, McGraw-Hill, New York, 1955.

Fillius, R. W., and C. E. McIlwain, Measurements of the Jovian radiation belts, J. Geophys. Res., 79, 3589, 1974.

Fujimoto, M., and A. Nishida, Monte Carlo simulation of energization of Jovian trapped electrons by recirculation, J. Geophys. Res., 95, 3841, 1990a.

Fujimoto, M., and A. Nishida, Energization and anisotropization of energetic electrons in the Earth's radiation belt by the recirculation process, J. Geophys. Res., 95, 4265, 1990 b.

Hood, L. L., Radiation diffusion in the Uranian radiation belts: Inferences from satellite absorption loss models, J. Geophys. Res., 94, 15,077, 1989.

Krimigis, S. M., T. P. Armstrong, W. I. Axford, A. F. Cheng, G. Gloeckler, D. C. Hamilton, E. P. Keath, L. J. Lanzerotti, and B. H. Mauk, The magnetosphere of Uranus: Hot plasma and radiation environment, Science, 233, 97, 1986

Lupton, J. E., and E. C. Stone, Measurements of electron detection efficiencies in solid state detectors, Nucl. Instrum. Methods, 98, $189,1972$.

Mauk, B. H., S. M. Krimigis, E. P. Keath, A. F. Cheng, T. P. Armstrong, L. J. Lanzerotti, G. Gloeckler, and D. C. Hamilton, The hot plasma and radiation environment of the Uranian magnetosphere, J. Geophys. Res., 92, 15,283, 1987.

McIlwain, C. E., Coordinates for mapping the distribution of magnetically trapped particles, J. Geophys. Res., 66, 3681, 1961.

McKibben, R. B., and J. A. Simpson, Charged particle diffusion and acceleration in Saturn's radiation belts, J. Geophys. Res., 85, $5773,1980$.

Mogro-Campero, A., and W. Fillius, The absorption of trapped particles by the inner satellites of Jupiter and the radial diffusion coefficient of particle transport, J. Geophys. Res., 81, 1289, 1976.

Nakada, M. P., High-energy electrons in the radiation belt, $J$. Geophys. Res., 68, 47, 1963.

Ness, N. F., M. H. Acuña, K. W. Behannon, L. F. Burlaga,
J. E. P. Connerney, R. P. Lepping, and F. M. Neubauer, Magnetic fields at Uranus, Science, 233, 85, 1986.

Nishida, A., Outward diffusion of energetic particles from the Jovian radiation belt, J. Geophys. Res., 81, 1771, 1976.

Paonessa, M. T., and A. F. Cheng, Satellite sweeping in offset, tilted dipole fields, J. Geophys. Res., 92, 1160, 1987.

Schulz, M., Jupiter's radiation belts, Space Sci. Rev., 23, 277, 1979.

Schulz, M., and L. J. Lanzerotti, Particle Diffusion in the Radiation Belts, Springer-Verlag, New York, 1974.

Selesnick, R. S., and E. C. Stone, Energetic electrons at Uranus: Bimodal diffusion in a satellite limited radiation belt, J. Geophys. Res., 96, 5651, 1991.

Sittler, E. C., Jr., K. W. Ogilvie, and R. Selesnick, Survey of electrons in the Uranian magnetosphere: Voyager 2 observations, J. Geophys. Res., 92, 15,263, 1987.

Stilwell, D. E., W. D. Davis, R. M. Joyce, F. B. McDonald, J. H. Trainor, W. E. Althouse, A. C. Cummings, T. L. Garrard, E. C. Stone, and R. E. Vogt, The Voyager Cosmic Ray Experiment, IEEE Trans. Nucl. Sci., NS-26(1), 513, 1979.

Stone, E. C., The physical significance and application of $L, B_{0}$, and $R_{0}$ to geomagnetically trapped particles, J. Geophys. Res., $68,4157,1963$.

Stone, E. C., and E. D. Miner, The Voyager 2 encounter with the Uranian system, Science, 233, 39, 1986.

Stone, E. C., R. E. Vogt, F. B. McDonald, B. J. Teegarden, J. H. Trainor, J. R. Jokipii, and W. R. Webber, Cosmic ray investigation for the Voyager missions: Energetic particle studies in the outer heliosphere-and beyond, Space Sci. Rev., 21, 355, 1977.

Stone, E. C., J. F. Cooper, A. C. Cummings, F. B. McDonald J. H. Trainor, N. Lal, R. McGuire, and D. L. Chenette, Energetic charged particles in the Uranian magnetosphere, Science, 233, 93 , 1986.

Thomsen, M. F., C. K. Goertz, and J. A. Van Allen, On determining magnetospheric diffusion coefficients from the observed effects of Jupiter's satellite Io, J. Geophys. Res., 82, 5541, 1977.

Van Allen, J. A., D. N. Baker, B. A. Randall, and D. D. Sentman, The magnetosphere of Jupiter as observed with Pioneer 10, 1, Instrument and principal findings, J. Geophys. Res., 79, 3559, 1974.

Van Allen, J. A., M. F. Thomsen, and B. A. Randall, The energetic charged particle absorption signature of Mimas, J. Geophys. Res., $85,5709,1980$.

J. F. Cooper, National Space Science Data Center, STX Suite 300, 7601 Ora Glen Drive, Greenbelt, MD 20770.

E. C. Stone, Downs Laboratory of Physics, California Institute of Technology, Pasadena, CA 91125.

(Received January 2, 1990;

revised November 16, 1990;

accepted November 21, 1990.) 